\title{
Kaon Condensation in Proto-Neutron Star Matter
}

\author{
Jose A. Pons ${ }^{1,2}$, Sanjay Reddy ${ }^{3}$, Paul J. Ellis ${ }^{4}$, Madappa Prakash ${ }^{1}$, and James M. Lattimer ${ }^{1}$ \\ ${ }^{1}$ Department of Physics \& Astronomy, SUNY at Stony Brook, Stony Brook, New York 11794-3800 \\ ${ }^{2}$ Departament d'Astronomia i Astrofísica, Universitat de València, E-46100 Burjassot, Spain \\ 3 Institute For Nuclear Theory, University of Washington, Seattle, WA 98195 \\ ${ }^{4}$ School of Physics $\&$ Astronomy, University of Minnesota, Minneapolis, MN 55455
}

(October 31, 2018)

\begin{abstract}
We study the equation of state of kaon-condensed matter including the effects of temperature and trapped neutrinos. Several different field-theoretical models for the nucleon-nucleon and kaon-nucleon interactions are considered. It is found that the order of the phase transition to a kaon-condensed phase, and whether or not Gibbs' rules for phase equilibrium can be satisfied in the case of a first order transition, depend sensitively on the choice of the kaon-nucleon interaction. To avoid the anomalous high-density behavior of previous models for the kaon-nucleon interaction, a new functional form is developed. For all interactions considered, a first order phase transition is possible only for magnitudes of the kaon-nucleus optical potential $\gtrsim 100 \mathrm{MeV}$. The main effect of finite temperature, for any value of the lepton fraction, is to mute the effects of a first order transition, so that the thermodynamics becomes similar to that of a second order transition. Above a critical temperature, found to be at least 30-60 MeV depending upon the interaction, the first order transition disappears. The phase boundaries in baryon density versus lepton number and baryon density versus temperature planes are delineated, which are useful in understanding the outcomes of protoneutron star simulations. We find that the thermal effects on the maximum gravitational mass of neutron stars are as important as the effects of trapped neutrinos, in contrast to previously studied cases in which the matter contained only nucleons or in which hyperons and/or quark matter were considered. Kaoncondensed equations of state permit the existence of metastable neutron stars, because the maximum mass of an initially hot, lepton-rich protoneutron star is greater than that of a cold, deleptonized neutron star. The large thermal effects imply that a metastable protoneutron star's collapse to a black hole could occur much later than in previously studied cases that allow metastable configurations.
\end{abstract}

PACS numbers(s): 13.15.+g, 13.75.Jz, 26.60.+c, 97.60.Jd 


\section{INTRODUCTION}

It is believed that a neutron star begins its life as a proto-neutron star (PNS) in the aftermath of a supernova explosion. The evolution of the PNS depends upon the star's mass, composition, and equation of state (EOS), as well as the opacity of neutrinos in dense matter. Previous studies [1] 3] have shown that the PNS may become unstable as it emits neutrinos and deleptonizes, so that it collapses into a black hole. The instability occurs if the maximum mass that the equation of state (EOS) of lepton-rich, hot matter can support is greater than that of cold, deleptonized matter, and if the PNS mass lies in between these two values. The condition for metastability is satisfied if "exotic" matter, manifested in the form of a Bose condensate (of negatively charged pions or kaons) or negatively charged particles with strangeness content (hyperons or quarks), appears during the evolution of the PNS.

Even if collapse to a black hole does not occur, the appearance of exotic matter might lead to a distinguishable feature in the PNS's neutrino signature (i.e., its neutrino light curve and neutrino energy spectrum) that is observable from current and planned terrestrial detectors. This was investigated recently by Pons et al. [1] who studied the evolution of a PNS in the case where hyperons appeared in the star during the latter stages of deleptonization. Although the possibility of black hole formation was first discovered in the context of kaon condensation in neutron star matter [2], a full dynamical calculation of a PNS evolution with consistent EOS and neutrino opacities in kaon condensed matter has not been performed so far. One of the objectives of this paper is to investigate $K^{-}$condensation in finite temperature matter, including the situation of trapped neutrinos in more detail. An impetus for this study is the recent suggestion that a mixed phase of kaon-condensed and normal matter might exist which could greatly affect the structure [5] and its neutrino opacity [6]. Another objective of our study is to identify differences in thermodynamic quantities such as the pressure, entropy or specific heat that might produce discriminating features in the star's neutrino emission. In separate works, we will examine neutrino interactions in kaon-condensed matter and neutrino signals from PNS evolution calculations in a consistent fashion.

Since we wish to isolate the aforementioned effects due to kaons in this paper, we deliberately exclude consideration of hyperons. Hyperons and kaons were considered together in Refs. [7] and [8]. Hyperons tend to delay the appearence of kaons in matter, especially if the $\Sigma^{-}$appears first. However, the $\Sigma^{-}$ couplings are not as well determined as those of the $\Lambda$ and even in this case the data are restricted to nuclear or subnuclear densities. Relatively small variations in the coupling constants can lead to a situation where the threshold density for the appearance of $\Sigma^{-}$particles is larger than that for kaons. These uncertainties remain unresolved; further hyper-nuclear experiments are needed to pin down their couplings.

The original investigations of kaon condensation in neutron star matter (e.g. Refs. [9 11] and its astrophysical conseqences [2, 12]) employed a chiral $S U(3)_{L} \times S U(3)_{R}$ model in which the kaon-nucleon interaction occurs directly via four point vertices. However, one can also employ an indirect, finite-range interaction which arises from the exchange of mesons. Several studies have been performed along these lines [5,7,8, 13, 14]. Ref. [7] found that the chiral and meson exchange approaches give similar results provided that the kaon-nucleon couplings are chosen to yield similar optical potentials in nuclear matter. Allowing kaons to interact via the exchange of mesons has the advantage that it is more consistent with the Waleckatype effective field-theoretical models usually used to describe nuclear matter [15]. In most studies of kaon condensation it has been found that the transition to a phase in which kaons condense is second order for modest values of the kaon optical potential, $U_{K}$, of order $-100 \mathrm{MeV}$. For magnitudes of $U_{K}$ well in excess of $100 \mathrm{MeV}$, however, the phase transition becomes first order in character. Even when the transition is first order, it is not always possible to satisfy Gibbs' criteria for thermal, chemical and mechanical 
equilibrium, so a Maxwell construction, which satisfies only thermal and mechanical equilibrium, was sometimes employed to construct the pressure-density relation.

Recently, Glendenning and Schaffner-Bielich (GS) [5] modified the meson exchange Lagrangian in such a way that the Gibbs criteria for thermal, chemical and mechanical equilibrium in a first order phase transition was possible. The extended mixed phase of kaon-condensed and normal matter which results produces a qualitative difference for the structure of a neutron star, since the EOS is softened over a wider region than in the case in which there is no mixed phase. This has implications for the mass-radius relation and the maximum mass, among other properties of the star.

In this paper, we investigate the phase transition involving kaon-condensed matter and its influence upon the equation of state. We find that the precise form assumed for the scalar interactions (particularly, their density dependence), both for baryon-baryon and kaon-baryon interactions, determines whether or not the transition is first or second order, and, in the case of a first order phase transition, establishes whether or not a Gibbs construction is possible. Since the form of the scalar interactions is not experimentally well constrained at present, we have explored several different models in this study of the effects of kaon condensation on the EOS and the structure of a PNS. For each model, we have performed a detailed study of the thermal properties which are summarized in terms of phase diagrams in the density-lepton content and density-temperature planes.

In Sec. II we present the various Lagrangians and derive exressions for the thermodynamic properties of each. We also develop the theoretical formalism necessary to describe baryons and kaon condensed matter in both the pure and mixed phases. This is followed by a discussion of the determination of the various coupling constants. Section III contains a comparison of the results for the EOS and for the structure of neutron stars for typical values of entropy and lepton content in a proto-neutron star as it evolves. Our conclusions and outlook for evolution of a proto-neutron simulation are presented in Sec. IV. In Appendix A, the extent of the correspondence between a meson exchange formalism and a chiral model to describe kaon condensation in matter is examined. The role of higher order kaon self-interactions in determining the order of the phase transition to a kaon condensed state is studied in Appendix B.

\section{THEORY}

\section{A. Nucleons and Leptons}

We begin with the well-known relativistic field theory model of Walecka 15 supplemented by nonlinear scalar self-interactions [16]. Here nucleons $(n, p)$ interact via the exchange of $\sigma-, \omega-$, and $\rho$-mesons. Explicitly, the Lagrangian is

$$
\begin{aligned}
\mathcal{L}_{\mathcal{N}}= & \sum_{n, p} \bar{N}\left(i \gamma^{\mu} \partial_{\mu}-g_{\omega} \gamma^{\mu} \omega_{\mu}-g_{\rho} \gamma^{\mu} \mathbf{b}_{\mu} \cdot \mathbf{t}-M^{*}\right) N+\frac{1}{2} \partial_{\mu} \sigma \partial^{\mu} \sigma-\frac{1}{2} m_{\sigma}^{2} \sigma^{2}-U(\sigma) \\
& -\frac{1}{4} \omega_{\mu \nu} \omega^{\mu \nu}+\frac{1}{2} m_{\omega}^{2} \omega_{\mu} \omega^{\mu}-\frac{1}{4} \mathbf{B}_{\mu \nu} \cdot \mathbf{B}^{\mu \nu}+\frac{1}{2} m_{\rho}^{2} \mathbf{b}_{\mu} \cdot \mathbf{b}^{\mu}
\end{aligned}
$$

where $N$ is the nucleon field, the $\rho$-meson field is denoted by $\mathbf{b}_{\mu}$ and the quantity $\mathbf{t}$ is the isospin operator which acts on the nucleons. Scalar self-couplings [16], which improve the descripton of nuclear matter at the equilibrium density, are included in the potential $U(\sigma)=(b M / 3)\left(g_{\sigma} \sigma\right)^{3}+(c / 4)\left(g_{\sigma} \sigma\right)^{4}$, with $M$ denoting the vacuum nucleon mass. The field strength tensors for the vector mesons are given by the expressions $\omega_{\mu \nu}=\partial_{\mu} \omega_{\nu}-\partial_{\nu} \omega_{\mu}$ and $\mathbf{B}_{\mu \nu}=\partial_{\mu} \mathbf{b}_{\nu}-\partial_{\nu} \mathbf{b}_{\mu}$. In the standard Walecka model the nucleon effective mass

$$
M_{G M}^{*}=M-g_{\sigma} \sigma
$$


(the label GM refers to the Glendenning-Moszkowski parameters [17] that we will use with this expression). We shall also study an alternative form due to Zimanyi and Moszkowski (labelled by ZM) [18]:

$$
\begin{aligned}
\mathcal{L}_{\mathcal{N}}= & \sum_{n, p}\left\{\left(1+\frac{g_{\sigma} \sigma}{M}\right) \bar{N}\left(i \gamma^{\mu} \partial_{\mu}-g_{\omega} \gamma^{\mu} \omega_{\mu}-g_{\rho} \gamma^{\mu} \mathbf{b}_{\mu} \cdot \mathbf{t}\right) N-\bar{N} M N\right\}+\frac{1}{2} \partial_{\mu} \sigma \partial^{\mu} \sigma-\frac{1}{2} m_{\sigma}^{2} \sigma^{2}-U(\sigma) \\
& -\frac{1}{4} \omega_{\mu \nu} \omega^{\mu \nu}+\frac{1}{2} m_{\omega}^{2} \omega_{\mu} \omega^{\mu}-\frac{1}{4} \mathbf{B}_{\mu \nu} \cdot \mathbf{B}^{\mu \nu}+\frac{1}{2} m_{\rho}^{2} \mathbf{b}_{\mu} \cdot \mathbf{b}^{\mu}
\end{aligned}
$$

By redefining the nucleon field, $N \rightarrow\left(1+g_{\sigma} \sigma / M\right)^{-\frac{1}{2}} N$, the Lagrangian can be written exactly in the form Eq. (四), but the nucleon effective mass becomes

$$
M_{Z M}^{*}=M\left(1+g_{\sigma} \sigma / M\right)^{-1} .
$$

For small values of $\sigma$ this is equivalent to the Walecka form. However the ZM effective mass has the property that, in the limit of large $\sigma, M_{Z M}^{*}$ remains positive whereas $M_{G M}^{*}$ can become negative [7, 19], which is unphysical.

In the mean field approximation the thermodynamic potential per unit volume for both Lagrangians is

$$
\frac{\Omega_{\mathcal{N}}}{V}=\frac{1}{2} m_{\sigma}^{2} \sigma^{2}+U(\sigma)-\frac{1}{2} m_{\omega}^{2} \omega_{0}^{2}-\frac{1}{2} m_{\rho}^{2} b_{0}^{2}-2 T \sum_{n, p} \int \frac{d^{3} k}{(2 \pi)^{3}} \ln \left(1+\mathrm{e}^{-\beta\left(E^{*}-\nu_{n, p}\right)}\right) .
$$

Here the inverse temperature is denoted by $\beta=1 / T, E^{*}=\sqrt{k^{2}+M^{* 2}}$ and the subscripts $G M$ or $Z M$ have been suppressed. The chemical potentials are given by

$$
\mu_{p}=\nu_{p}+g_{\omega} \omega_{0}+\frac{1}{2} g_{\rho} b_{0} \quad ; \quad \mu_{n}=\nu_{n}+g_{\omega} \omega_{0}-\frac{1}{2} g_{\rho} b_{0}
$$

Note that in a rotationally invariant system only the time components of the vector fields contribute to Eq. (5) and for the isovector field only the $\rho^{0}$ component contributes. The contribution of antinucleons is not significant for the thermodynamics of interest for a PNS and is ignored.

Using $\Omega_{\mathcal{N}}$, the thermodynamic quantities can be obtained in the standard way. The nucleon pressure is $P_{\mathcal{N}}=-\Omega_{\mathcal{N}} / V$, and the number density $n_{n, p}$ and the energy density $\varepsilon_{\mathcal{N}}$ are given by

$$
\begin{aligned}
n_{n, p} & =2 \int \frac{d^{3} k}{(2 \pi)^{3}} f_{F}\left(E^{*}-\nu_{n, p}\right), \\
\varepsilon_{\mathcal{N}} & =\frac{1}{2} m_{\sigma}^{2} \sigma^{2}+U(\sigma)-\frac{1}{2} m_{\omega}^{2} \omega_{0}^{2}-\frac{1}{2} m_{\rho}^{2} b_{0}^{2}+2 \sum_{i=n, p} \int \frac{d^{3} k}{(2 \pi)^{3}} E^{*} f_{F}\left(E^{*}-\nu_{i}\right),
\end{aligned}
$$

where the Fermi distribution function $f_{F}(x)=\left(e^{\beta x}+1\right)^{-1}$. The entropy density is then given by $S_{\mathcal{N}}=$ $\beta\left(\varepsilon_{\mathcal{N}}+P_{\mathcal{N}}-\sum_{N} \mu_{N} n_{N}\right)$

The contribution from the leptons and antileptons is adequately given by its non-interacting form, since their interactions give negligible contributions [21]. Thus the thermodynamic potential per unit volume of the leptons and antileptons is:

$$
\frac{\Omega_{L}}{V}=-\sum_{\ell} T g_{\ell} \int \frac{d^{3} k}{(2 \pi)^{3}}\left[\ln \left(1+\mathrm{e}^{-\beta\left(e_{\ell}-\mu_{\ell}\right)}\right)+\ln \left(1+\mathrm{e}^{-\beta\left(e_{\ell}+\mu_{\ell}\right)}\right)\right],
$$

where $g_{\ell}$ and $\mu_{\ell}$ denote the degeneracy and the chemical potential, respectively, of leptons of species $\ell$. The degeneracy $g_{\ell}$ is 2 for electrons and muons and it is 1 for neutrinos of a given species. Since the 
star is in chemical equilibrium with respect to the weak processes $p+\ell^{-} \leftrightarrow n+\nu_{\ell}$, where the lepton $\ell$ is either an electron or a muon, the chemical potentials obey $\mu_{\mu}-\mu_{\nu_{\mu}}=\mu_{e}-\mu_{\nu_{e}}=\mu_{n}-\mu_{p}$. If there are no neutrinos trapped in the star the neutrino chemical potentials $\mu_{\nu_{i}}$ are zero or, equivalently, the total neutrino concentration $Y_{\nu}=0$, where we define the concentration for particle $i$ to be $Y_{i}=n_{i} /\left(n_{n}+n_{p}\right)$. The pressure, density and energy density of the leptons are obtained from Eq. (8) in standard fashion.

\section{B. Kaons}

The two kaon Lagrangians of the meson-exchange type which have been previously suggested (in Refs. [1.:7] and [5:8], respectively), can both be written in the form

$$
\mathcal{L}_{K}=\partial_{\mu} K^{+} \partial^{\mu} K^{-}-\alpha K^{+} K^{-}+i X^{\mu}\left(K^{+} \partial_{\mu} K^{-}-K^{-} \partial_{\mu} K^{+}\right)
$$

where $K^{ \pm}$denote the charged kaon fields and we have defined the combined vector field

$$
X_{\mu}=g_{\omega K} \omega_{\mu}+g_{\rho K} b_{\mu}
$$

with $\omega_{\mu}$ and $b_{\mu}$ denoting $\omega$ and the $\rho^{0}$ fields, respectively; $g_{\omega K}$ and $g_{\rho K}$ are coupling constants. Since only the time components of the vector fields survive, in practice only $X_{0}$ is non-zero. The two Lagrangians differ in the forms chosen for the quantity $\alpha$. Both have the standard vacuum mass term, but the interaction terms differ. Specifically, Knorren, Prakash and Ellis (KPE) [7] take

$$
\alpha_{K P E}=m_{K ; K P E}^{* 2}=m_{K}^{2}-g_{\sigma K} m_{K} \sigma
$$

with $m_{K}$ denoting the vacuum kaon mass, while Glendenning and Schaffner-Bielich (GS) [5] choose

$$
\alpha_{G S}=m_{K ; G S}^{* 2}-X_{\mu} X^{\mu}=\left(m_{K}-\frac{1}{2} g_{\sigma K} \sigma\right)^{2}-X_{\mu} X^{\mu}
$$

Note that the coupling constant $g_{\sigma K}$ is defined here to be twice that defined in GS. A similar remark applies to the $\rho N N$ coupling constant $g_{\rho}$. It is remarkable, as pointed out in Appendix A, that to leading order in the kaon condensate intensity, the equations obtained with the chiral Kaplan-Nelson [9] Lagrangian at zero temperature agree precisely with those from the KPE Lagrangian.

To see the significance of the term involving the vector fields in Eq. (12), consider the invariance of the Lagrangian under the transformation $K^{ \pm} \rightarrow K^{ \pm} e^{ \pm i \xi}$. This allows the conserved kaon current density to be identified as

$$
J_{\mu}=i\left(K^{+} \partial_{\mu} K^{-}-K^{-} \partial_{\mu} K^{+}\right)+2 X_{\mu} K^{+} K^{-}
$$

Now for the combined GS Lagrangian, $\mathcal{L}_{\mathcal{N}}+\mathcal{L}_{K}$, the equation of motion for the omega field is

$$
\partial^{\nu} \omega_{\nu \mu}+m_{\omega}^{2} \omega_{\mu}=g_{\omega} \sum_{n, p} \bar{N} \gamma_{\mu} N-g_{\omega K} J_{\mu}
$$

Since the nucleon current $\sum_{n, p} \bar{N} \gamma_{\mu} N$ is conserved, as is the kaon current $J_{\mu}$, taking the divergence of Eq. (14) immediately yields $\partial^{\mu} \omega_{\mu}=0$ (and similarly for the $\rho$ field). This is the required condition for a vector field [20] so as to reduce the number of components from four to three. On the other hand, $\alpha_{K P E}$ does not contain an $X_{\mu} X^{\mu}$ term, so that the kaon current does not appear on the right hand side of Eq. (14). 
At the mean field level, however, where the vector fields are constants, any derivative is necessarily zero so that the divergence condition is automatically satisfied.

For the coupling of the kaon fields to the scalar $\sigma$ field, KPE use a linear coupling, whereas GS have an additional quadratic term. There is little guidance on the form that should be used to generate the kaon effective mass so the choice is somewhat arbitrary, although, as we shall see, it can significantly affect the thermodynamics. Both the KPE and GS choices lead to problems for sufficiently large values of the $\sigma$ field; in one case the effective mass becomes imaginary, in the other it becomes negative. We are therefore led to consider a third form in the spirit of the ZM model for nucleons. For specificity we start with the GS Lagrangian which can be written $\mathcal{L}_{K}=D_{\mu} K^{+} D^{\mu *} K^{-}-m_{K ; G S}^{* 2} K^{+} K^{-}$in terms of a covariant derivative $D_{\mu}=\partial_{\mu}+i X_{\mu}$, and replace it by

$$
\mathcal{L}_{K}^{\prime}=\left(1+\frac{g_{\sigma K} \sigma}{2 m_{K}}\right)^{2} D_{\mu} K^{+} D^{\mu *} K^{-}-m_{K}^{2} K^{+} K^{-}
$$

We observe that the form of $\mathcal{L}_{K}^{\prime}$ above is one of many possibilities. Making the transformation $K^{ \pm} \rightarrow$ $\left(1+\frac{1}{2} g_{\sigma K} \sigma / m_{K}\right)^{-1} K^{ \pm}$and noting that $\sigma$ is a constant mean field, the kaon Lagrangian can be put in the form of Eq. (9) with

$$
\alpha_{T W}=m_{K ; T W}^{* 2}-X_{\mu} X^{\mu}=m_{K}^{2}\left(1+\frac{g_{\sigma K} \sigma}{2 m_{K}}\right)^{-2}-X_{\mu} X^{\mu}
$$

The label $T W$ denotes "this work". While Eqs. (11), (12) and (16) all give $m_{K}^{*} \simeq m_{K}-\frac{1}{2} g_{\sigma K} \sigma$ for small $\sigma$, they differ at order $\sigma^{2}$ and beyond, i.e., for large values of $\sigma$.

The kaon partition function at finite temperature can be obtained for a Lagrangian of the form (9) by generalizing the procedure outlined in Kapusta [21]. First, we transform to real fields $\phi_{1}$ and $\phi_{2}$,

$$
K^{ \pm}=\left(\phi_{1} \pm i \phi_{2}\right) / \sqrt{2}
$$

and determine the conjugate momenta

$$
\pi_{1}=\partial_{0} \phi_{1}-X_{0} \phi_{2} \quad ; \quad \pi_{2}=\partial_{0} \phi_{2}+X_{0} \phi_{1}
$$

The Hamiltonian density is $\mathcal{H}_{K}=\pi_{1} \partial_{0} \phi_{1}+\pi_{2} \partial_{0} \phi_{2}-\mathcal{L}_{K}$, and the partition function of the grand canonical ensemble can then be written as the functional integral

$$
Z_{K}=\int\left[d \pi_{1}\right]\left[d \pi_{2}\right] \int_{\text {periodic }}\left[d \phi_{1}\right]\left[d \phi_{2}\right] \exp \left\{\int_{0}^{\beta} d \tau \int d^{3} x\left(i \pi_{1} \frac{\partial \phi_{1}}{\partial \tau}+i \pi_{2} \frac{\partial \phi_{2}}{\partial \tau}-\mathcal{H}_{K}\left(\pi_{i}, \phi_{i}\right)+\mu J_{0}\left(\pi_{i}, \phi_{i}\right)\right)\right\}
$$

Here the fields obey periodic boundary conditions in the imaginary time $\tau=i$, namely $\phi_{i}(\mathbf{x}, 0)=\phi_{i}(\mathbf{x}, \beta)$, where $\beta=1 / T$. The chemical potential associated with the conserved kaon charge density is denoted by $\mu$ and chemical equilibrium in the reaction $e^{-} \leftrightarrow K^{-}+\nu_{e}$ requires that $\mu=\mu_{n}-\mu_{p}=\mu_{e}-\mu_{\nu_{e}}=\mu_{\mu}-\mu_{\nu_{\mu}}$.

The Gaussian integral over momenta in Eq. (19) is easily performed. Next the fields are Fourier decomposed according to

$$
\phi_{1}=f \theta \cos \zeta+\sqrt{\frac{\beta}{V}} \sum_{n, \mathbf{p}} e^{i\left(\mathbf{p} \cdot \mathbf{x}+\omega_{n} \tau\right)} \phi_{1, n}(\mathbf{p}) \quad ; \quad \phi_{2}=f \theta \sin \zeta+\sqrt{\frac{\beta}{V}} \sum_{n, \mathbf{p}} e^{i\left(\mathbf{p} \cdot \mathbf{x}+\omega_{n} \tau\right)} \phi_{2, n}(\mathbf{p}),
$$


where the first term describes the condensate, so that in the second term $\phi_{1,0}(\mathbf{p}=0)=\phi_{2,0}(\mathbf{p}=0)=0$. The pion decay constant $f$ has been inserted so that the condensate angle $\theta$ is dimensionless. The Matsubara frequency $\omega_{n}=2 \pi n T$. The partition function can then be written

$$
\begin{aligned}
Z_{K} & =N \int \prod_{n, \mathbf{p}}\left[d \phi_{1, n}(\mathbf{p})\right]\left[d \phi_{2, n}(\mathbf{p})\right] e^{S}, \quad \text { where } \\
S & =\frac{1}{2} \beta V(f \theta)^{2}\left(\mu^{2}+2 \mu X_{0}-\alpha\right)-\frac{1}{2} \sum_{n, \mathbf{p}}\left(\phi_{1,-n}(-\mathbf{p}), \phi_{2,-n}(-\mathbf{p})\right) \mathbf{D}\left(\begin{array}{c}
\phi_{1, n}(\mathbf{p}) \\
\phi_{2, n}(\mathbf{p})
\end{array}\right), \\
\mathbf{D} & =\beta^{2}\left(\begin{array}{cc}
\omega_{n}^{2}+p^{2}+\alpha-2 \mu X_{0}-\mu^{2} & 2\left(\mu+X_{0}\right) \omega_{n} \\
-2\left(\mu+X_{0}\right) \omega_{n} & \omega_{n}^{2}+p^{2}+\alpha-2 \mu X_{0}-\mu^{2}
\end{array}\right) .
\end{aligned}
$$

$N$ is a normalization constant. We define the $K^{ \pm}$energies according to

$$
\omega^{ \pm}(p)=\sqrt{p^{2}+\alpha+X_{0}^{2}} \pm X_{0}
$$

so that the three approaches give

$$
\begin{aligned}
& \omega_{K P E}^{ \pm}(p)=\sqrt{p^{2}+m_{K ; K P E}^{* 2}+X_{0}^{2}} \pm X_{0} \\
& \omega_{G S}^{ \pm}(p)=\sqrt{p^{2}+m_{K ; G S}^{* 2}} \pm X_{0} \\
& \omega_{T W}^{ \pm}(p)=\sqrt{p^{2}+m_{K ; T W}^{* 2}} \pm X_{0} .
\end{aligned}
$$

Using the definition (22) and suppressing the explicit dependence of $\omega^{ \pm}$on $p$, the determinant of $\mathbf{D}$ is

$$
\operatorname{det} \mathbf{D}=\beta^{4}\left[\omega_{n}^{2}+\left(\omega^{-}-\mu\right)^{2}\right]\left[\omega_{n}^{2}+\left(\omega^{+}+\mu\right)^{2}\right]
$$

giving

$$
\frac{\Omega_{K}}{V}=-\frac{\ln Z_{K}}{\beta V}=\frac{1}{2}(f \theta)^{2}\left(\alpha-2 \mu X_{0}-\mu^{2}\right)+\frac{1}{2 \beta V} \sum_{n, \mathbf{p}} \ln \operatorname{det} \mathbf{D},
$$

where the normalization constant $N$ has been dropped since it is irrelevant to the thermodynamics. Performing the sum over $n$ and neglecting the zero-point contribution, which contributes only beyond the mean field approach and in any case is small [22], we obtain the grand potential for the kaon sector:

$$
\frac{\Omega_{K}}{V}=\frac{1}{2}(f \theta)^{2}\left(\alpha-2 \mu X_{0}-\mu^{2}\right)+T \int_{0}^{\infty} \frac{d^{3} p}{(2 \pi)^{3}}\left[\ln \left(1-e^{-\beta\left(\omega^{-}-\mu\right)}\right)+\ln \left(1-e^{-\beta\left(\omega^{+}+\mu\right)}\right)\right] .
$$

The kaon pressure, $P_{K}=-\Omega_{K} / V$, and the kaon number density is easily found to be

$$
n_{K}=(f \theta)^{2}\left(\mu+X_{0}\right)+n_{K}^{T H} \quad \text { where } \quad n_{K}^{T H}=\int \frac{d^{3} p}{(2 \pi)^{3}}\left[f_{B}\left(\omega^{-}-\mu\right)-f_{B}\left(\omega^{+}+\mu\right)\right]
$$

and the Bose occupation probability $f_{B}(x)=\left(e^{\beta x}-1\right)^{-1}$. The kaon energy density is

$$
\varepsilon_{K}=\frac{1}{2}(f \theta)^{2}\left(\alpha+\mu^{2}\right)+\int \frac{d^{3} p}{(2 \pi)^{3}}\left[\omega^{-}(p) f_{B}\left(\omega^{-}-\mu\right)+\omega^{+}(p) f_{B}\left(\omega^{+}+\mu\right)\right]
$$

and the kaon entropy density is $S_{K}=\beta\left(\varepsilon_{K}+P_{K}-\mu n_{K}\right)$. 


\section{Equations of Motion}

It is useful first to define the quantity

$$
A_{K}^{T H}=\int \frac{d^{3} p}{(2 \pi)^{3}}\left(p^{2}+\alpha+X_{0}^{2}\right)^{-\frac{1}{2}}\left[f_{B}\left(\omega^{-}-\mu\right)+f_{B}\left(\omega^{+}+\mu\right)\right] .
$$

Then the mean $\omega, \rho$ and $\sigma$ fields, as well as the condensate amplitude $\theta$, determined by extremizing the total grand potential $\Omega_{\text {total }}=\Omega_{\mathcal{N}}+\Omega_{L}+\Omega_{K}$, can be written

$$
\begin{aligned}
& m_{\omega}^{2} \omega_{0}=g_{\omega}\left(n_{p}+n_{n}\right)-g_{\omega K}\left\{\mu(f \theta)^{2}+n_{K}^{T H}-X_{0} A_{K}^{T H}-\frac{1}{2}\left[(f \theta)^{2}+A_{K}^{T H}\right] \frac{\partial \alpha}{\partial X_{0}}\right\} \\
& m_{\rho}^{2} b_{0}=\frac{1}{2} g_{\rho}\left(n_{p}-n_{n}\right)-g_{\rho K}\left\{\mu(f \theta)^{2}+n_{K}^{T H}-X_{0} A_{K}^{T H}-\frac{1}{2}\left[(f \theta)^{2}+A_{K}^{T H}\right] \frac{\partial \alpha}{\partial X_{0}}\right\} \\
& m_{\sigma}^{2} \sigma=-\frac{d U(\sigma)}{d \sigma}-2 \frac{\partial M^{*}}{\partial \sigma} \sum_{n, p} \int \frac{d^{3} k}{(2 \pi)^{3}} \frac{M^{*}}{E^{*}} f_{F}\left(E^{*}-\nu_{n, p}\right)-\frac{1}{2}\left[(f \theta)^{2}+A_{K}^{T H}\right] \frac{\partial \alpha}{\partial \sigma} \\
& \theta\left(\mu^{2}+2 \mu X_{0}-\alpha\right)=\theta\left[\mu-\omega^{-}(0)\right]\left[\mu+\omega^{+}(0)\right]=0 .
\end{aligned}
$$

The derivative $\partial \alpha / \partial X_{0}$ is zero for the KPE case and $-2 X_{0}$ for the GS and TW cases. The derivatives with respect to the $\sigma$ field are

$$
\begin{aligned}
& \frac{\partial M_{G M}^{*}}{\partial \sigma}=-g_{\sigma} \quad ; \quad \frac{\partial M_{Z M}^{*}}{\partial \sigma}=-g_{\sigma}\left(\frac{M_{Z M}^{*}}{M}\right)^{2} \\
& \frac{\partial \alpha_{K P E}}{\partial \sigma}=-g_{\sigma K} m_{K} \quad ; \quad \frac{\partial \alpha_{G S}}{\partial \sigma}=-g_{\sigma K} m_{K ; G S}^{*} \quad ; \quad \frac{\partial \alpha_{T W}}{\partial \sigma}=-g_{\sigma K} \frac{\left(m_{K ; T W}^{*}\right)^{3}}{m_{K}^{2}} .
\end{aligned}
$$

Note that the last of Eqs. (30) yields either $\theta=0$ (no condensate) or the condition for a condensate to exist. Since $\mu$ is positive here, we only have the possibility of a $K^{-}$condensate with $\mu=\omega^{-}(0)$. Note also that the contribution of the condensate to the kaon pressure $P_{K}$ vanishes, as it should.

The remaining condition to be imposed is that neutron star matter must be charge neutral. For a single phase this implies

$$
n_{p}-n_{K}-n_{e}-n_{\mu}=0
$$

where $n_{e}$ and $n_{\mu}$ are the net negative lepton number densities. The mixed phase thermodynamics is discussed below.

The sum of the nucleon and kaon energy densities can be simplified somewhat by using the equations of motion. This gives

$$
\begin{aligned}
\varepsilon_{\mathcal{N}}+\varepsilon_{K}= & \frac{1}{2} m_{\sigma}^{2} \sigma^{2}+U(\sigma)+\frac{1}{2} m_{\omega}^{2} \omega_{0}^{2}+\frac{1}{2} m_{\rho}^{2} b_{0}^{2}+2 \sum_{n, p} \int \frac{d^{3} k}{(2 \pi)^{3}} E^{*} f_{F}\left(E^{*}-\nu_{n, p}\right)+(f \theta)^{2}\left(\alpha-\frac{1}{2} X_{0} \frac{\partial \alpha}{\partial X_{0}}\right) \\
& +X_{0} n_{K}^{T H}-X_{0} A_{K}^{T H}\left(X_{0}+\frac{1}{2} \frac{\partial \alpha}{\partial X_{0}}\right)+\int \frac{d^{3} p}{(2 \pi)^{3}}\left[\omega^{-}(p) f_{B}\left(\omega^{-}-\mu\right)+\omega^{+}(p) f_{B}\left(\omega^{+}+\mu\right)\right] .
\end{aligned}
$$




\section{Mixed Phase Thermodynamics}

In the theory discussed above there are two independent chemical potentials, which we can take to be $\mu_{n}$ and $\mu$, each connected with a conserved charge, the baryon number and charge of the system, respectively. Glendenning [23] pointed out that in the presence of a first order phase transition, conservation laws must be globally, not locally, imposed, if possible, in the mixed phase region. A Maxwell construction would have been appropriate had there been just a single conserved charge. However, relaxing the condition of local charge neutrality does not guarantee that the model Lagrangian, solved in the mean field approximation, will provide a description of the mixed phase, which is only possible if the Gibbs criteria can be satisifed. A simple, yet general, procedure to check if the Gibbs criteria can be fulfilled by a specific model is discussed in Appendix B.

Denoting the phase containing a condensate with a subscript $\theta$, and the phase without a condensate with $\theta=0$, the total pressures in the two phases must be equal

$$
P_{\theta=0}\left(\mu_{n}, \mu, T\right)=P_{\theta}\left(\mu_{n}, \mu, T\right) .
$$

Each of the chemical potentials is the same in the two phases. If the volume fraction of the non-condensed phase is $\chi$, then global conservation of charge requires

$$
\chi\left[n_{p}-n_{K}-n_{e}-n_{\mu}\right]_{\theta=0}+(1-\chi)\left[n_{p}-n_{K}-n_{e}-n_{\mu}\right]_{\theta}=0 .
$$

The densities of the individual species in the mixed phase are evident here. The total energy density is the weighted sum of the two phases

$$
\varepsilon=\chi \varepsilon_{\theta=0}\left(\mu_{n}, \mu, T\right)+(1-\chi) \varepsilon_{\theta}\left(\mu_{n}, \mu, T\right) .
$$

The total entropy density is obtained through a similar equation.

\section{E. Coupling Constants}

In the effective Lagrangian approach adopted here, knowledge of two distinct sets of coupling constants, one parametrizing the nucleon-nucleon interactions, and one parametrizing the kaon-nucleon interactions, are required for numerical computations. These are associated with the exchange of $\sigma, \omega$ and $\rho$ mesons. We consider each of these in turn.

\section{Nucleon Couplings}

The nucleon-meson coupling constants are determined by adjusting them to reproduce the properties of equilibrium nuclear matter at $T=0$. The properties used are the saturation density, $n_{0}$, the binding energy/particle, $E_{A}$, the symmetry energy coefficient, $a_{\text {sym }}$, the compression modulus, $K$, and the Dirac effective mass at saturation, $M^{*}$. Not all of these quantities are precisely known and the values we choose are listed in Table I. For completeness, we list the equations needed to obtain the coupling constants, assuming that the scalar self-coupling has the form $U(\Phi)=(b M / 3) \Phi^{3}+(c / 4) \Phi^{4}$, where $\Phi=g_{\sigma} \sigma$. From the equation of motion for the $\omega_{0}$ field and the fact that the pressure is zero at saturation density in nuclear matter, the value of $g_{\omega} / m_{\omega}$ is given by 


$$
\frac{g_{\omega}^{2}}{m_{\omega}^{2}}=\frac{M-E_{A}-E_{F}^{*}}{n_{0}}
$$

where $E_{F}^{*}=\sqrt{k_{F}^{2}+M^{* 2}}$ and $n_{0}=2 k_{F}^{3} /\left(3 \pi^{2}\right)$. The $\rho$ meson coupling constant can be determined for a given symmetry energy through the relation

$$
\frac{g_{\rho}^{2}}{m_{\rho}^{2}}=\frac{4}{n_{0}}\left(2 a_{s y m}-\frac{k_{F}^{2}}{3 E_{F}^{*}}\right)
$$

An expression involving the compression modulus can be deduced by differentiating the $\sigma$ equation of motion:

$$
\left(\frac{g_{\sigma}^{2}}{m_{\sigma}^{2}}\right)^{-1}=-\left[f\left(\Phi_{0}\right)\right]^{2}\left\{\frac{9 n_{0} M^{* 2}}{E_{F}^{* 2}\left[K+9\left(E_{A}+E_{F}^{*}-M\right)\right]-3 k_{F}^{2} E_{F}^{*}}-3\left(\frac{n_{0}}{E_{F}^{*}}-\frac{n_{s}}{M^{*}}\right)\right\}+n_{s} \frac{d f}{d \Phi_{0}}-\frac{d^{2} U\left(\Phi_{0}\right)}{d \Phi_{0}^{2}}
$$

Here $\Phi_{0}$, the value of $\Phi$ at saturation density, is obtained directly from the Dirac effective mass. The function $f\left(\Phi_{0}\right)=-d M^{*} / d \Phi_{0}$ depends on the particular expression used for the effective mass. The scalar density $n_{s}=\left(M^{*} / \pi^{2}\right)\left\{k_{F} E_{F}^{*}-M^{* 2} \ln \left[\left(k_{F}+E_{F}^{*}\right) / M^{*}\right]\right\}$. The $\sigma$ equation of motion at saturation can be written in the form

$$
\Phi_{0}^{2} \frac{d^{2} U\left(\Phi_{0}\right)}{d \Phi^{2}}=6\left[n_{0}\left(\frac{1}{2} E_{F}^{*}+E_{A}-M\right)+n_{s}\left(\frac{1}{2} M^{*}+\Phi_{0} f\right)\right]
$$

which together with Eq. (39) allows the $\sigma$ coupling to be obtained. Finally the constants appearing in the scalar self-coupling $U(\Phi)$ are determined from:

$$
\begin{aligned}
& c=\frac{1}{\Phi_{0}^{2}}\left[\frac{d^{2} U\left(\Phi_{0}\right)}{d \Phi_{0}^{2}}+\frac{2 m_{\sigma}^{2}}{g_{\sigma}^{2}}-2 n_{s} \frac{f}{\Phi_{0}}\right] \\
& b=\frac{1}{2 M \Phi_{0}}\left[\frac{d^{2} U\left(\Phi_{0}\right)}{d \Phi_{0}^{2}}-3 c \Phi_{0}^{2}\right] .
\end{aligned}
$$

The constants determined in this way are given in Table I. Note that in principle the potential should be bounded from below for large values of the $\sigma$ field requiring $c$ to be positive; this is the case for the $\mathrm{ZM}$ model.

\section{Kaon Couplings}

In order to investigate the effect of a kaon condensate on the EOS in high-density matter, the kaonmeson coupling constants have to be specified. Empirically known quantities can be used to determine these constants, but it should be borne in mind that laboratory experiments give information only about kaon-nucleon interaction in free space or in nearly isospin symmetric nuclear matter. On the other hand, the physical setting in this work is matter in the dense interiors of neutron stars which has a different composition and spans a wide range of densities (up to $\sim 8 n_{0}$ ). Therefore, kaon-meson couplings as determined from experiments might not be appropriate to describe the kaon-nucleon interaction in neutron star matter, and the particular choices of coupling constants should be regarded as parameters that have a range of uncertainty. 
With the above caveats in mind, we now examine the relationship between the optical potential of a single kaon in infinite nuclear matter and the kaon-meson couplings in our Lagrangian. Lagrange's equation for an $s$-wave $K^{-}$with a time dependence $K^{-}=k^{-}(\mathbf{x}) e^{-i E t}$, where $E$ is the asymptotic energy, defines the optical potential [24] for our Lagrangian (9) according to

$$
\begin{aligned}
{\left[\nabla^{2}+E^{2}-m_{K}^{2}\right] k^{-}(\mathbf{x}) } & =\left[-2 X_{0} E+\alpha-m_{K}^{2}\right] k^{-}(\mathbf{x}) \\
& \equiv 2 m_{K} U_{K} k^{-}(\mathbf{x}) .
\end{aligned}
$$

In nuclear matter, $b_{0}=0$, so for a kaon with zero momentum $\left(E=m_{K}\right)$ the optical potential is

$$
U_{K}=\frac{\alpha-m_{K}^{2}}{2 m_{K}}-g_{\omega K} \omega_{0} .
$$

Utilizing the functional forms for $\alpha$ in Eqs. (11), (12), and (16), the optical potentials for the KPE, GS and TW models are easily obtained. For the KPE case this may be written exactly as

$$
U_{K}^{K P E}=-\frac{1}{2} g_{\sigma K} \sigma-g_{\omega K} \omega_{0},
$$

whereas for the GS and TW cases there are higher order corrections in addition to the terms linear in the fields. We choose $g_{\omega K}$ to be $g_{\omega} / 3$ and $g_{\rho K}$ to be $g_{\rho} / 2$ on the basis of simple quark and isospin counting arguments. Note that this value for $g_{\omega K}$ is also suggested by comparison to the chiral approach (see Ref. [7] and Appendix A) and it leads to a $-48.5 \mathrm{MeV}$ contribution to the optical potential. The total optical potential is shown in Table II for various choices of the $\sigma$ coupling. The linear form Eq. (44), exact for $\mathrm{KPE}$, is an accurate fit to the the GS and TW cases for moderate values of the optical potential. For orientation, chiral models suggest that the magnitude of the optical potential is at most $120 \mathrm{MeV}$ [7], while fits to kaonic atom data have been reported with values in the range 50-200 MeV [25 28]. We note that Glendenning and Schaffner-Bielich [5] label their results according to values of the optical potential obtained in the linear approximation (henceforth, $U_{K}^{\operatorname{lin}}$ ). In order to make an apposite comparison with their results, we will parametrize the kaon coupling for each model simply by specifying the value of $U_{K}^{\operatorname{lin}}=U_{K}^{K P E}$.

\section{RESULTS AND DISCUSSION}

\section{A. Zero temperature case}

The effects of kaon condensation on the EOS are more pronounced at zero temperature than at finite temperature, since the fraction of thermally excited kaons increases with temperature relative to the fraction of kaons residing in the condensate. We therefore begin by examining results for the zero temperature case. We have considered two different nucleon Lagrangians, GM and ZM, and three different kaon Lagrangians, KPE, GS and TW. Below densities of about $0.5 n_{0}$, matter is composed of neutron-rich nuclei immersed in a neutron sea. For this regime, we use the potential model results of Negele and Vautherin [29] in the range $0.001<n<0.08 \mathrm{fm}^{-3}$ and those of Baym, Bethe, and Pethick [30] for $n<0.001 \mathrm{fm}^{-3}$. For cold stars, the EOS in this regime has little effect on maximum masses or stellar radii. Furthermore, since the entropy in the stellar mantle $\left(n<n_{0}\right)$ is quickly radiated away in neutrinos, the EOS in this regime does not substantially affect the results of this paper.

In Fig. 1, we compare the pressures for the different nucleon and kaon Lagrangians as a function of baryon density, $n_{B}=n_{n}+n_{p}$. The solid lines show results for both the pure nucleon and kaon condensed 
phases with no attempt to enforce the Gibbs conditions of chemical and mechanical equilibrium. In all cases, a first order phase transition is found to occur, as long as the magnitude of the optical potential $\left|U_{K}^{\text {lin }}\right|=\frac{1}{2} g_{\sigma K} \sigma+g_{\omega K} \omega$ is in excess of $100 \mathrm{MeV}$. Where possible, the pressure in the mixed phase obtained by imposing Gibbs' criteria for mechanical and chemical equilibrium is shown as a dashed line. For the GM+KPE, ZM+KPE and ZM+GS models it was not possible to satisfy Gibb's criteria, despite the occurrence of a first order phase transition for large enough $\left|U_{K}^{\text {lin }}\right|$. The reason for this is connected with the form of the kaon Lagrangian, as discussed below. We also point out in Appendix B that non-linear kaon self-interactions lead to a second order, rather than a first order, transition.

The qualitative similarity of the results shown in Fig. [f for the different nuclear Lagrangians enables us to simplify our analysis by allowing us to focus on three, rather than six, possible Lagrangian combinations. For a given kaon Lagrangian, fairly similar results can be obtained with different nuclear Lagrangians by making relatively small shifts in the kaon optical potential $U_{K}^{\text {lin }}$. The following discussion will therefore focus on the three cases GM+KPE, GM+GS and ZM+TW. The case GM+KPE is chosen to compare with the results of KPE, the case GM+GS is chosen to compare with the results of GS, and the case $\mathrm{ZM}+\mathrm{TW}$ demonstrates the usefulness of Lagrangians in which anomalous values of effective masses are implicitly eliminated. The results for the model GM+GS shown here and elsewehere in this paper are identical to those found by GS for the same interactions. Note that in all models considered, the phase transition is second order in nature for moderately low values of the optical potential.

In Figs. 2 and 0 , the density dependence of the scalar, vector, and iso-vector fields, the electron chemical potential $\mu_{e}=\mu=\mu_{n}-\mu_{p}$, the condensate amplitude $\theta$, and the nucleon and kaon effective masses are displayed in the pure nucleon and kaon condensed phases, ignoring any possible mixed phase for the present. For the optical potential chosen, $U_{K}^{\text {lin }}=-120 \mathrm{MeV}$, a first order phase transition occurs in all three cases. After the onset of condensation a rapid change in the behavior of the electron chemical potential and some of the field strengths is seen to occur. The differences in the variation of the scalar $\left(g_{\sigma K} \sigma\right)$ and isovector $\left(-g_{\rho K} b_{0}\right)$ fields between the models are particularly illuminating. For GM+KPE, the scalar field exhibits a relatively rapid increase with density after the onset of condensation. This in turn causes both the nucleon and kaon effective masses to drop rapidly with density. In fact, for sufficiently large density, the GM+KPE kaon effective mass vanishes (see Fig. 3). The variations of the effective masses in the models GM+GS and ZM+TW are more moderate. The variation of the isovector field with density, which in large part controls the variation of the electron chemical potential $\mu$ and hence the electron concentration, is also more dramatic in the case of GM+KPE than in the GM+GS and ZM+TW models. Notice that in the KPE model it goes to zero for asymptotic densities (this follows from Eq. (30)), so that the proton and neutron abundances become equal. This does not occur for the other two cases considered here. Finally, it is worth noting that in all three models the condensate amplitude rises rapidly once the threshold density is reached.

We turn now to a discussion of the results obtained by imposing Gibbs' criteria for mechanical and chemical equilibrium at zero temperature. In Fig. 目, we show the chemical potentials associated with the two conserved charges, charge and baryon number, as functions of each other, for the model GM+GS for a kaon optical potential of $U_{K}^{\text {lin }}=-120 \mathrm{MeV}$. Quantities associated with the pure nucleon phase, Phase I, are shown as solid lines here and in subsequent figures. Phase II refers to the high-density phase in which nucleons and the kaon condensate are in equilibrium, and quantities associated with it are shown as dashed lines. Both phases, I and II, coexist in the mixed-phase region which is displayed as a dotted line. This figure illustrates the way a mixed phase is built from the two pure phases. For electron chemical potentials below the solid curve, matter is positively charged in phase I. A similar interpretation of positive or negative charge for $\mu$ below or above the dashed curve is not possible, since two different types of particles, kaons 
and leptons, can furnish charge. In other words, a decrease in $\mu$, or, equivalently, the number of electrons, does not necessarily lead to a positive net charge in phase II. For $\mu_{n} \leq 1165 \mathrm{MeV}$, only phase I with nucleons and leptons are present. For $\mu_{n} \leq 1310 \mathrm{MeV}$, a mixed phase of positively charged phase I and negatively charged phase II obeying the Gibbs' conditions (34) is favored. Qualitatively, a similar situation is encountered in the construction of the mixed phase for the ZM+TW model, but the mixed phase region is quite small. As noted earlier, however, it was not possible to satisfy Gibbs' criteria for models with the kaon Lagrangian KPE.

In Fig. 5 we show the individual charge densities of phase I and II in the mixed phase, as a function of baryon density. The dotted curve in this figure shows the volume fraction of phase I. The results are for the GM+GS model with $U_{K}^{\text {lin }}=-120 \mathrm{MeV}$ (upper panel) and for the ZM+TW model with $U_{K}^{\text {lin }}=-140$ $\mathrm{MeV}$ (lower panel). Near the lower threshold, matter in phase I is very slightly positively charged and occupies most of the volume. As the density increases, the volume fraction of phase I, $\chi$, decreases and its charge density increases. Note that the negative charge density of matter in phase II at the lower transition point, $\approx 0.5 \mathrm{fm}^{-3}$, and the positive charge density of matter in phase $\mathrm{I}$ at the higher transition point, $\approx 1 \mathrm{fm}^{-3}$, are rather large in the case of GM+GS compared to the case ZM+TW. This is due to the stronger density dependence of the scalar and isovector densities in the former case. Note also that a first order transition allows for the existence of a very dense and nearly isospin symmetric matter in the mixed phase.

In Figs. 6 and 7, we show the magnitudes of the various fields, the electron chemical potential, the nucleon and kaon effective masses, and the condensate amplitude for the GM+GS and ZM+TW models, respectively. Both models show the same qualitative behavior. At the lower phase boundary, in which phase II just begins to appear, the scalar field in phase II is much larger than in phase I and the condensate amplitude $\theta$ in phase II takes a large value which decreases with increasing $n_{B}$ through the mixed-phase region. Thus, the effective masses of both kaons and nucleons in phase II are much smaller than in phase I. The densities demarking the mixed phase region and its overall extent are dependent upon the interaction models, and upon the assumed values of the kaon optical potentials, here taken to be $-140 \mathrm{MeV}$ in the case of $\mathrm{GM}+\mathrm{GS}$ and $-160 \mathrm{MeV}$ in the case of ZM+TW. The region in density over which the mixed phase extends is much smaller in the latter case, chiefly due to the more moderate behavior of the scalar interaction with density variations in this case.

It is instructive to compare the behavior of the two models at the threshold of the mixed phase region. Phase I will have a net small positive charge and a volume proportion $\chi$ close to 1 (see Fig. 5). This has to be counterbalanced by a large net negative charge in phase II since it is weighted by the small proportion $(1-\chi)$. Focusing on phase II, the condensate condition for the models GM+GS and ZM+TW from the last of Eqs. (30) is

$$
\mu+X_{0}=m_{K}^{*}
$$

and the kaon number density, which has to be large, is

$$
n_{K}=(f \theta)^{2}\left(\mu+X_{0}\right) .
$$

In order to ensure that $n_{K}>0$, the quantity $\left(\mu+X_{0}\right)$, and hence $m_{K}^{*}$, has to be positive definite. In the ZM+TW model the kaon effective mass is relatively large so that $X_{0}$ is positive and therefore $\theta$ is relatively small. On the other hand in the GM+GS model $m_{K}^{*}$ is quite small so that $X_{0}$ is negative and $\theta$ has to be large. The negative value of $X_{0}=g_{\omega K} \omega_{0}+g_{\rho K} b_{0}$ implies a large negative value of

$$
g_{\rho K} b_{0}=\frac{g_{\rho K} g_{\rho}}{2 m_{\rho}^{2}}\left(n_{p}-n_{n}-\frac{2 g_{\rho K}}{g_{\rho}} n_{K}\right),
$$


which is clearly sensitive to the value of $g_{\rho K}$. In fact, if this coupling is reduced by more than about $15 \%$ from our chosen value it is no longer possible to satisfy the Gibbs criteria. By comparing the pure phase results in Figs. 2 and 3 with the mixed phase results of Figs. 6 and 0 , it is clear that substantial modifications of the various fields are required to satisfy Gibbs' criteria.

We examine now the KPE model for which it was not possible to satifsfy the Gibbs' criteria. In this case, Eq. (11) and the last of Eqs. (30) leads to the condensate condition

$$
\mu\left(\mu+2 X_{0}\right)=\left(m_{K}^{*}\right)^{2},
$$

whereas the functional form of the number density of kaons is identical to that in Eq. (46). Eq. (48) differs in important ways from Eq. (45). For the KPE model, even if $\mu+2 X_{0}$ is positive, $\mu$ has the proclivity to turn negative for large $\mu_{n}$ (or equivalently, for large baryon densities), leading to $\left(m_{K}^{*}\right)^{2}<0$ or imaginary values of the kaon effective mass $m_{K}^{*}$. This may be seen in Fig. 8 where we show the electron chemical potential $\mu$ as a function of the (negative) charge density in pure phase II for a typical value of the neutron chemical potential $\mu_{n}=1250 \mathrm{MeV}$. It is now possible to understand qualitatively why a mixed phase cannot occur in the case of the kaon Lagrangian KPE. In comparison with the GM+GS and $\mathrm{ZM}+\mathrm{TW}$ models, a distinctive feature of the KPE model is that $\mu$ decreases rapidly with the (negative) charge density. In constructing a mixed phase, we are attempting to balance the positive charge in phase I with the negative charge in the dense phase II in which the electron chemical potential, and hence the charge content in leptons, is rapidly decreasing towards zero. The balance never occurs, hence the failure to meet the Gibbs' criteria. In terms of compositions, the GS or TW Lagrangians introduce negative charges in matter by increasing the number density of kaons, while keeping the electron density nearly constant or even slightly increasing with the charge density. The KPE Lagrangian, however, rapidly substitutes electrons by kaons, which is detrimental to meeting the Gibbs' criteria. For these reasons, we will concentrate on results with the other two kaon Lagrangians in the remainder of this paper.

The influence of the condensate on neutron star structure (at zero temperature) is shown in Fig. 9 in which the gravitational mass is displayed as a function of the star's central baryon number density (left panel) and its radius (right panel). For the models shown, the transition is first order and Gibbs equations for mechanical and chemical equilibrium are utilized. For all cases shown the central densities of the maximum mass stars lie in the mixed phase. The effects of the condensate are more evident in the case of the GM+GS model in which the mixed phase occurs over a wider region of density than in the ZM+TW model. When the effects of the softening induced by the occurrence of the condensate are large, the limiting mass and the radius at the limiting mass are reduced significantly from their values when the condensate is absent. Note, however, that the softening effects are limited by the constraint that the maximum mass must exceed that of the binary pulsar PSR 1913+16, $1.442 \mathrm{M}_{\odot}$. In the case of GM+GS, this constraint limits $\left|U_{K}^{\text {lin }}\right|$ to be smaller than about $125 \mathrm{MeV}$. In such a case, the minimum radius achieved is not as small as in the case $U_{K}^{\text {lin }}=-140 \mathrm{MeV}$, as shown in Fig. 9. The radii of stars with masses less than $1.2 \mathrm{M}_{\odot}$ are not affected by the choice of the kaon Lagrangian or the kaon optical potential, since the condensation threshold is not reached in these cases.

\section{B. Comparison with other works}

The density dependence of $m_{K}^{*} / m_{K},-U_{K}$ and $\omega_{K}$ have been investigated in other works [26 28,31.32], but for the most part either in isospin symmetric nuclear matter or pure neutron matter. In general, our results for $m_{K}^{*} / m_{K}$ with $-U_{K}^{\text {lin }}=80 \mathrm{MeV}$ are consistent with those of Refs. 27,28] (for an appropriate 
comparison, our results are to be compared with results obtained without in-medium pion contributions in Ref. [27]) and those of Ref. [26] for nuclear matter at both $n / n_{0}=1$ and 3. There is a relatively small change produced in going from nuclear matter to beta-equilibrated neutron star matter to pure neutron matter for the quantities $m_{K}^{*} / m_{K}$ and $-U_{K}$. Note that a direct comparison of the real parts of the optical potentials between different calculations must also account for the fact that in obtaining fits to data, the imaginary parts are often found to be as large as the real parts, which indicates fragmentation of strength in the quasi-particle spectral function.

Relatively larger variations are found in the kaon energies in matter with varying amounts of isospin as can be seen from Fig. 10. In this figure, the top panel provides a comparison of results for beta-equilibrated neutron-star matter for the GM+KPE, GM+GS, and ZM+TW models, respectively, for values of $-U_{K}^{\text {lin }}$ at the extreme ends considered here, namely, 80 and $120 \mathrm{MeV}$. The bottom panel shows results for the $\mathrm{ZM}+\mathrm{TW}$ model for $-U_{K}^{\mathrm{lin}}=80 \mathrm{MeV}$, for pure neutron matter, neutron-star matter, and isospin symmetric nuclear matter, respectively. At nuclear density where the models are calibrated, $\omega$ decreases by about a few $\mathrm{MeV}$ in going from pure neutron matter to neutron star matter and by about a few tens of $\mathrm{MeV}$ in going from neutron star matter to nuclear matter. With increasing density, these differences become progressively larger. This trend is chiefly due to the behavior of the vector fields in matter with different amounts of isospin.

At this time, our results for the density dependence of $\omega$ can be compared with those of the potential models in Refs. 31,32. For values of $-U_{K}^{\text {lin }}$ near the lower end of the range we explored, in the neighborhood of $80 \mathrm{MeV}$, the behavior of $\omega$, for example, is quite similar to the potential model results. As the authors in Refs. [31, 32] indicated, kaon condensation may be unlikely in this case. However, the relevant comparision must also include the electron chemical potential $\mu_{e}$, since the density where $\omega=\mu_{e}$ determines the onset of kaon condensation. As demonstrated in Ref. [33], the behavior of $\mu_{e}$ for neutron star matter at high densities is determined by the density dependence of the nuclear symmetry energy (see also a similar discussion in Ref. [31]). Potential model calculations (see, for example Ref. [34]) tend to have a relatively weak density dependence of the symmetry energy, which generally results in an onset of kaon condensation that is at a rather large density. In field-theoretical and Dirac-Brueckner-Hartree-Fock [35] models, however, the symmetry energy varies relatively rapidly with density. These lead to smaller densities where kaon condensation occurs, for a given behavior of the kaon energy $\omega$. Furthermore, the calculations of Ref. [31] have been performed only for pure neutron matter which further enhaces the values of $\omega$ and discourages kaon condensation. In addition, as $\left|U_{K}^{\text {lin }}\right|$ is increased in magnitude in field-theoretical models, the role of kaons increases and $\omega$ becomes progressively smaller as a function of density. Nevertheless, the lack of effective constraints at high density preclude choosing any model over another at this time.

In summary, choosing values of $-U_{K}^{\text {lin }}$ near the lower end of the range we explored either lead to a second order phase transition or no transition at all in a neutron star, in which case the gross properties of the star are relatively unaffected from the case without kaons. On the other hand, values near the higher end of this range lead to a first order phase transition at a relatively low density, depending on the form of the interaction chosen, and a more pronounced effect on the star. Our aim has been to provide benchmark calculations in which both possibilities are entertained in order to consider their impact on thermodynamics and their astrophysical implications.

\section{Finite temperature case}

We now investigate results at finite temperature and values of the lepton content characteristic of those

likely to be encountered in the evolution of a PNS. We choose three representative sets of PNS conditions 
which correspond to: the initial conditions within a PNS (entropy/baryon $s=1$, trapped neutrinos with a lepton fraction $\left.Y_{L}=0.35\right)$, a time after several seconds when the interior is maximally heated $(s=2$, no trapped neutrinos so $\left.Y_{\nu}=0\right)$, and a very late time when the PNS has cooled $\left(s=0, Y_{\nu}=0\right.$-identical to the zero temperature case discussed above). For a detailed explanation of the evolution of a cooling PNS see Pons et al. [四].

The contribution of the nucleons to the entropy per baryon $s_{\mathcal{N}} \equiv S_{\mathcal{N}} / n_{B}$, with $n_{B}=n_{n}+n_{p}$ denoting the total nucleon density, in degenerate situations $\left(T / E_{F_{i}} \ll 1\right)$ can be written

$$
s_{\mathcal{N}}=\pi^{2} T \frac{\sum_{i=n, p} k_{F, i} \sqrt{M^{*^{2}}+k_{F_{i}}^{2}}}{\sum_{i=n, p} k_{F, i}^{3}}
$$

where $M^{*}$ and $k_{F, i}$ are the effective mass and the Fermi momentum of species $i$, respectively. For the temperatures of interest here, and particularly with increasing density, the above relation provides an accurate representation of the exact results for entropies per baryon even up to $s_{\mathcal{N}}=s_{n}+s_{p} \simeq 2$. The behavior with density of both the Fermi momenta and the effective mass controls the temperatures for a fixed $s_{\mathcal{N}}$.

For kaons it is straightforward to show that the contribution to the entropy from $K^{+}$mesons can be ignored since it is exponentially suppressed in comparison to the $K^{-}$contribution. For the latter, keeping the leading temperature dependence of the simplest approximation scheme for bosons given in Ref. [36], the kaon entropy per baryon is

$$
s_{K} \equiv \frac{S_{K}}{n_{B}}=\left[\frac{5}{4}(2-y)-\psi\right] \frac{n_{K}^{T H}}{n_{B}} \quad \text { where } \quad \psi T=\mu+X_{0}-\sqrt{\alpha+X_{0}^{2}},
$$

and $y$ is determined from $\psi$ by solving the equation

$$
\psi=1-y+\ln y \text {. }
$$

Below the kaon condensation threshold as the temperature becomes very small $\psi \rightarrow-\infty$ so $y \rightarrow 0$. Above the kaon condensation threshold the last of Eqs. (30) implies that $\psi=0$ in which case $y=1$. This simple approximation provides quite an accurate account of the kaon entropy per baryon which is fairly small for the scenarios examined here since it involves just the thermal contribution and the condensate plays no role. The total entropy per baryon $s_{\text {tot }}=s_{\mathcal{N}}+s_{K}+\left(S_{e}+S_{\mu}+S_{\nu}\right) / n_{B}$ also includes the lepton contributions; $s_{\text {tot }}$ is dominated, however, by $s_{\mathcal{N}}$.

In Figs. 11 and 12, the relative concentrations of various particles are displayed versus baryon number density for our three PNS conditions in the cases GM+GS and ZM+TW, respectively. The cases shown allow the Gibbs equations to be solved, and the boundaries of the mixed phase regions are indicated by vertical lines. The effect of finite temperature is to allow the existence of $\mu^{-}$and $K^{-}$particles at all densities, although kaons become relatively abundant only within the mixed phase region. In the third set of diagrams, trapped neutrinos are present at all densities and the appearance and abundances of the negatively charged particles $\mu^{-}$and $K^{-}$are suppressed. Furthermore, the critical density for kaon condensation is shifted to higher density.

In Fig. 13 the pressure is displayed as a function of baryon number density for these two Lagrangians and the three PNS conditions. Two choices for the kaon optical potential are shown to highlight differences between cases in which kaons condense in second or first order phase transitions. The reduction of the pressure when kaons condense is obvious. For conditions in which the phase transition is first order, the result of applying the Gibbs conditions and the result of assuming pure phases (thin line) are both shown. 
The application of the Gibbs conditions leads to further softening of the pressure over a wider density range. In the case of model $\mathrm{ZM}+\mathrm{TW}$, a first order phase transition occurs only for very low temperatures and low neutrino concentrations.

In Fig. 14 we show the matter temperature as a function of the baryon density for these two Lagrangians for the two PNS conditions with $s>0$ (the kaon optical potentials are as in the previous figure). The appearance of the kaon condensate generally leads to a reduction in specific heat which is indicated by the abrupt temperature increase which persists to high densities. In the case of first order transitions, applying the Gibbs conditions leads to a further enhancement of the temperature in the mixed phase regime. This behavior is in marked contrast to the case in which additional fermionic degrees of freedom, such as hyperons or quarks, are excited [i] causing the temperature to drop and the specific heat of the matter to be increased. The latter follows from Eq. (49) where, in the absence of any variation of $M^{*}$, a system with more components at a given baryon density has a smaller temperature than a system with fewer components (recall that $\sum_{i} Y_{i}=1$ ). In the present case the dropping of the effective mass is the dominant effect and this leads to larger temperatures.

Figure 19 shows the phase diagram of kaon condensed matter, for the case GM+GS with $U_{K}^{\text {lin }}=-120$ $\mathrm{MeV}$. The left panel displays results for zero temperature in the density-lepton concentration plane. The dashed lines show the minimum lepton concentration allowed at zero temperature (with $Y_{\nu}=0$ ) for each density. Note that the minimum lepton concentration increases with density until the phase transition begins; above this density, the minimum lepton concentration decreases with increasing density. Also note that the phase transition to a kaon-condensed phase is pushed to higher densities when trapped neutrinos are present. This implies that in the initial PNS core material, in which $Y_{L} \approx 0.35-0.4$ and the central density is less than 3.5 times the nuclear saturation density, a kaon condensate phase likely does not exist. However, as neutrinos leak from the star the transition density decreases and a kaon condensate eventually forms. The right panel displays results in the density versus temperature plane, assuming no trapped neutrinos $\left(Y_{\nu}=0\right)$.

The phase diagram for kaon condensed matter for the case ZM+TW with $U_{K}^{\text {lin }}=-140 \mathrm{MeV}$ is shown in Fig. 16; the results are qualitatively similar to the GM+GS case in which $U_{K}^{\mathrm{lin}}=-120 \mathrm{MeV}$ in Fig. 15. This is understandable from the perspective that the actual optical potential for these two models are nearly the same. The boundary between phases I and the mixed-phase region are nearly the same for the two cases. The major difference is the much smaller extent of the mixed-phase region for the case $\mathrm{ZM}+\mathrm{TW}$.

Note that for both cases the density at which the phase transition begins is relatively independent of temperature, so that the heating which initially occurs in the PNS has little effect on the eventual appearance of a kaon condensate. Also note that the density range of the mixed phase decreases with increasing temperature, and the mixed phase persists to high temperatures. It appears that the mixed phase exists up to temperatures exceeding $60 \mathrm{MeV}$, for the case GM+GS and $U_{K}^{\operatorname{lin}}=-120 \mathrm{MeV}$, or 30 $\mathrm{MeV}$ for the case $\mathrm{ZM}+\mathrm{TW}$ with $U_{K}^{\mathrm{lin}}=-140 \mathrm{MeV}$. It becomes increasingly difficult to determine the properties of a mixed phase near the temperature at which it disappears.

In Fig. 17 the gravitational mass is plotted as a function of central baryon number density for these models. Results are shown for our three PNS conditions which correspond to snapshots of the PNS evolution. The initial configuration (dotted curves) has the largest maximum mass. The progression to the dashed and solid curves indicates the evolution with time and we see that the maximum masses decrease. The effect of temperature upon the structure of the PNS is significant. Thermal kaons play a significant role here, since the net negative charge they contribute to the system partially inhibits the appearance of the condensate which allows hot neutrino-free stars to reach higher masses than cold stars. 
The net decrease in maximum mass during the evolution for either case is seen to be of order $0.2-0.3 \mathrm{M}_{\odot}$. Thus there is an appreciable range of masses for the PNS which will result in metastability with the star ultimately collapsing to a black hole. The central density of the maximum mass, zero temperature star is smaller for the GM+GS case than for the ZM+TW case. This is in spite of the apparently "softer" GM+GS equation of state in which the kaon condensed mixed-phase region extends over a wider density range. Ultimately, the smaller maximum mass of the GM+GS EOS leads to a smaller central density at the maximum mass.

\section{SUMMARY AND OUTLOOK}

In this work, we have studied the equation of state of matter, incorporating the possible presence of a kaon condensate, and including the effects of trapped neutrinos and finite temperatures. The calculation of the neutrino spectra of different flavors emitted from a proto-neutron star as it evolves from a hot, lepton-rich state to a cold, neutrino-poor state requires the knowledge of the equation of state of matter at temperatures up to about 50-60 MeV and lepton fraction up to about 0.4. Since the nucleon-nucleon and kaon-nucleon interactions at high density are relatively poorly understood, we explored several possible field-theoretical models in both sectors. These models are distinguished by the form of the assumed scalar (and in some cases vector) interactions which chiefly determine the density dependences of the nucleon and kaon effective masses. These models produce significantly different high density behavior of the EOS, even though the kaon-meson couplings in these models are calibrated to give the same the kaon-nucleus optical potential in nuclear matter.

The principal findings of our studies at zero temperature were:

1. The order of the phase transition between pure nucleonic matter and a phase containing a kaon condensate depends sensitively on the choice of the kaon-nucleon interaction.

2. In one case we studied (KPE), although a first-order phase transition resulted, it was not possible to satisfy Gibbs' rules for phase equilibrium which would have produced a mixed phase. We performed a detailed analysis of this situation and found that scalar, and to a lesser extent the isovector, interactions that vary rapidly with density were chiefly responsible for this failure. This was confirmed by developing a new kaon-nucleon interaction (TW) with more moderate variations in the scalar density and the kaon effective mass in which the Gibbs' criteria in a first order phase transition would be satisfied. The extent of the mixed phase region was thereby reduced. The significance of the new kaon-nucleon interaction (TW) we developed is that it avoids the anomalous behavior for the kaon effective mass that occurs in previous models (KPE, GS) at very high density. Near the low-density boundary of a mixed phase region, the kaon condensed phase appears with large density, too large for the KPE interaction to produce physically acceptable effective masses. We also made detailed comparisons with earlier work which used the GS form for the scalar interactions.

3. In all models considered (KPE, GS and TW), a first-order phase transition occurs only for large values of the kaon-nucleus optical potential; moderate values generally produce a second order phase transition.

In the meson exchange models studied here, only linear kaon self-interactions were considered. In the case of a first order phase transition, the condensate amplitude was found to be rather large at the lowdensity boundary of the mixed phase. We therefore explored the effect of non-linear kaon self-interactions 
guided by the chiral model in Appendix B. We found that introducing higher order interactions, using the lowest order chiral Lagrangian, results in a second order, rather than a first order, phase transition. Whether this behavior persists when more general higher order operators in the chiral expansion are considered remains an open question.

At finite temperatures, we find the effects of condensation, in general, are less pronounced than at zero temperature. For moderate values of the optical potential, when the phase transition is first order at zero temperature, kaon condensation eventually becomes a second order phase transition at high enough temperatures, whether or not neutrino trapping is considered. The temperature at which this occurs is in the range of 30-60 MeV, depending upon interactions. For the cases at finite temperatures in which the transition is first order, its thermodynamics (such as the pressure-density relation) becomes effectively similar to that of a second order phase transition. This is because of the existence of thermal kaons and because of nucleonic thermal effects. The condensate is suppressed, and moved to higher densities, both by the existence of trapped neutrinos and by finite temperatures. Compared to earlier works, the new aspects of our work are:

1. The delineation of the phase boundaries in the baryon density versus lepton number and baryon density versus temperature planes. This is helpful to anticipating the possible outcome in a full PNS simulation. In particular, the critical temperatures above which the mixed phase disappears are above 30 and $60 \mathrm{MeV}$, depending upon the interaction. This has implications for the temperature dependence of the surface energies, and for the melting temperatures, of the droplets in the mixed phase.

2. The finding that thermal effects on the maximum gravitational mass of neutron stars are comparable to the effects induced by the trapped neutrino content. This is in stark contrast to previously studied cases in which nucleons-only matter, or matter containing hyperons, were considered. Furthermore, compared to equations of state previously studied that allow metastable protoneutron stars, those containing hyperons or quark matter, the maximum mass does not significantly decrease during the deleptonization of the protoneutron star because of these thermal effects. Only after the temperature in the protoneutron star significantly decreases does the maximum mass appreciably fall. This implies that the possible collapse of a metastable protoneutron star to a black hole occurs during the late stages of cooling, after several tens of seconds, rather than during the late stages of deleptonization, which is somewhat earlier.

\section{ACKNOWLEDGEMENTS}

The support of the U.S. Department of Energy under contract numbers DOE/DE-FG02-87ER-40317 (JAP and JML), DOE/DE-FG06-90ER40561 (SR), DOE/DE-FG02-88ER-40328 (PJE), and DOE/DEFG02-88ER-40388 (MP) is acknowledged. J. Pons also gratefully acknowledges research support from the Spanish DGCYT grant PB97-1432, and thanks J.A. Miralles for useful discussions.

\section{APPENDIX A: MESON EXCHANGE VERSUS CHIRAL MODELS}

In this Appendix, we examine the conditions under which there exists a close correspondence between a meson exchange model and the chiral $S U(3)_{L} \times S U(3)_{R}$ approach of Kaplan and Nelson [9]. Such a correspondence is most easily established for the zero temperature case by setting the scalar self-coupling 
terms, i.e., $U(\sigma)=0$. Specializing to the case where the only baryons are nucleons and using the Walecka Lagrangian for the nucleons, it was shown in Ref. [1] that the chiral thermodynamic potential per unit volume can be written

$$
\begin{aligned}
\frac{\Omega_{\mathcal{N}}+\Omega_{K}}{V}= & \frac{1}{2} m_{\sigma}^{2} \sigma^{\prime 2}-\frac{1}{2} m_{\omega}^{2} \omega_{0}^{\prime 2}-\frac{1}{2} m_{\rho}^{2} b_{0}^{\prime 2}+2 \sum_{n, p} \int \frac{d^{3} k}{(2 \pi)^{3}}\left(E_{n, p}^{*}-\nu_{n, p}\right) \Theta\left(\nu_{n, p}-E_{n, p}^{*}\right) \\
& +2 m_{K}^{2} f^{2} \sin ^{2} \frac{1}{2} \theta-\frac{1}{2} \mu^{2} f^{2} \sin ^{2} \theta
\end{aligned}
$$

where the primes on the meson fields distinguish them from those used previously and $\Theta$ is the Heaviside step function. The nucleon effective masses are

$$
\begin{aligned}
& M_{n}^{*}=M-g_{\sigma} \sigma^{\prime}+\left(2 a_{2}+4 a_{3}\right) m_{s} \sin ^{2} \frac{1}{2} \theta \\
& M_{p}^{*}=M-g_{\sigma} \sigma^{\prime}+\left(2 a_{1}+2 a_{2}+4 a_{3}\right) m_{s} \sin ^{2} \frac{1}{2} \theta .
\end{aligned}
$$

We employ the values suggested by Politzer and Weise [10], namely $a_{1} m_{s}=-67 \mathrm{MeV}\left(m_{s}\right.$ is the strange quark mass) and $a_{2} m_{s}=+134 \mathrm{MeV} . a_{3} m_{s}$ is usually taken to lie in the range -134 to $-310 \mathrm{MeV}$. If we ignore the fairly small effect of $a_{1} m_{s}$ here and in the kaon-nucleon sigma term, $\Sigma^{K N}=-\frac{1}{2}\left(a_{1}+2 a_{2}+4 a_{3}\right) m_{s}$, we can write

$$
\begin{aligned}
& M_{n}^{*} \simeq M_{p}^{*} \simeq M^{*}=M-g_{\sigma} \sigma^{\prime}-2 \Sigma^{K N} \sin ^{2} \frac{1}{2} \theta \equiv M-g_{\sigma} \sigma \\
& E_{n, p}^{*} \simeq E^{*}=\sqrt{k^{2}+M^{* 2}} .
\end{aligned}
$$

As well as redefining the scalar field, we can redefine the chiral vector fields entering the chemical potentials:

$$
\begin{aligned}
& \mu_{n}=\nu_{n}+g_{\omega} \omega_{0}^{\prime}-\frac{1}{2} g_{\rho} b_{0}^{\prime}-\mu \sin ^{2} \frac{1}{2} \theta \equiv \nu_{n}+g_{\omega} \omega_{0}-\frac{1}{2} g_{\rho} b_{0} \\
& \mu_{p}=\nu_{p}+g_{\omega} \omega_{0}^{\prime}+\frac{1}{2} g_{\rho} b_{0}^{\prime}-2 \mu \sin ^{2} \frac{1}{2} \theta \equiv \nu_{p}+g_{\omega} \omega_{0}+\frac{1}{2} g_{\rho} b_{0} .
\end{aligned}
$$

Substituting in Eq. (52) we find

$$
\begin{aligned}
\frac{\Omega_{\mathcal{N}}+\Omega_{K}}{V}= & \frac{1}{2} m_{\sigma}^{2} \sigma^{2}-\frac{1}{2} m_{\omega}^{2} \omega_{0}^{2}-\frac{1}{2} m_{\rho}^{2} b_{0}^{2}+2 \sum_{n, p} \int \frac{d^{3} k}{(2 \pi)^{3}}\left(E^{*}-\nu_{n, p}\right) \Theta\left(\nu_{n, p}-E^{*}\right) \\
& +2 f^{2} \sin ^{2} \frac{1}{2} \theta\left\{m_{K}^{2}-\frac{m_{\sigma}^{2} \Sigma^{K N} \sigma}{g_{\sigma} f^{2}}-\frac{\mu}{4 f^{2}}\left(\frac{3 m_{\omega}^{2} \omega_{0}}{g_{\omega}}+\frac{2 m_{\rho}^{2} b_{0}}{g_{\rho}}\right)-\mu^{2} \cos ^{2} \frac{1}{2} \theta\right\} \\
& +\frac{1}{2} \sin ^{4} \frac{1}{2} \theta\left\{\left(\frac{2 m_{\sigma} \Sigma^{K N}}{g_{\sigma}}\right)^{2}-\mu^{2}\left(\frac{9 m_{\omega}^{2}}{4 g_{\omega}^{2}}+\frac{m_{\rho}^{2}}{g_{\rho}^{2}}\right)\right\}
\end{aligned}
$$

If we expand this in powers of $\theta$ and retain only the lowest order $\theta^{2}$ term, the last term in Eq. (56) does not contribute and our thermodynamic potential is exactly of the form given by Eqs. (5) and (26) for the meson exchange model provided that the $\alpha_{K P E}$ expression is used. In order for the correspondence to be exact, the parameters for the $\sigma$ and $\omega$ meson need to obey

$$
\frac{g_{\sigma} g_{\sigma K}}{m_{\sigma}^{2}}=\frac{\Sigma^{K N}}{m_{K} f^{2}} \quad ; \quad \frac{g_{\omega} g_{\omega K}}{m_{\omega}^{2}}=\frac{3}{8 f^{2}} .
$$

These are precisely the conditions found in Ref. [7] for the optical potentials of the chiral and meson exchange models to be the same in nuclear matter. The relation involving the $\omega$ meson couplings is quite well obeyed with our parameters. In addition, for the $\rho$ meson, 


$$
\frac{g_{\rho} g_{\rho K}}{m_{\rho}^{2}}=\frac{1}{4 f^{2}}
$$

This indicates that $\left(g_{\rho} g_{\rho K}\right) /\left(g_{\omega} g_{\omega K}\right) \simeq \frac{2}{3}$, a condition which is not well obeyed by the parameters used here or in other works. Given that the chiral and meson exchange thermodynamic potentials can be put into precise correspondence to lowest order in $\theta^{2}$, it follows that the equations of motion and the thermodynamics will be identical to this order.

If scalar self-coupling terms are included, $U(\sigma) \neq 0$, then the transition from the chiral to the meson exchange approach will couple higher powers of the $\sigma$ field to the kaon condensate (in the braces in Eq. (56)). It will also introduce higher order terms. These additional contributions may not be negligible so the correspondence between the two approaches becomes less precise.

\section{APPENDIX B: HIGHER ORDER KAON SELF-INTERACTIONS}

Our findings in Appendix A naturally raise the question of whether it is sufficient to work at order $\theta^{2}$, involving only linear kaon self-interactions, in the meson exchange models. It clearly will be sufficient at the low-density onset of a second order phase transition where $\theta$ is small. On the other hand, for a first order phase transition, the value of $\theta$ is large at the low-density onset of the mixed phase, particularly for the GS model. It is therefore interesting to explore the effect of non-linear kaon self-interactions guided by the chiral model.

The order of the phase transition (in the mean field approximation) is determined by the behavior of the thermodynamic potential, $\Omega(\theta)$, at fixed chemical potentials. A first order transition, with a mixed phase, is possible only if there exists some value of $\mu_{n}$ for which $\Omega(\theta)$ exhibits two degenerate minima. At the critical density corresponding to the low-density onset of the mixed phase, the $\theta=0$ phase should be a local minimum which is degenerate with a minimum at some finite $\theta=\theta_{c}$. In the vicinity of the critical density, the $\theta=0$ phase is nearly charge neutral (with an infinitesimal excess of positive charge and a volume fraction close to 1 which balances the negative charge in the kaon phase which has an infinitesimal volume fraction). This requirement enables us to determine the electron chemical potential at the critical density by charge neutrality.

We focus on the GM+GS model for which the thermodynamic potential of nucleons and kaons was given in Eqs. (5) and (26); the contribution due to leptons is ignored since it does not contain any $\theta$ dependence at fixed $\mu$. At zero temperature with a kaon optical potential $U_{K}^{\text {lin }}=-120 \mathrm{MeV}$, this model predicts a first order phase transition in the vicinity of $\mu_{n}=\mu_{c} \simeq 1160 \mathrm{MeV}$, as can be deduced from Fig. 18 where $\Omega(\theta)-\Omega(\theta=0)$ is plotted as a function of $\theta$. The thermodynamic potential for the model GM+GS is shown as the solid curve labelled $\Omega_{2}$. It clearly shows two minima, one at $\theta=0$ and the other at $\theta \simeq 2$. The latter corresponds to a kaon number density $n_{K} \sim 1 \mathrm{fm}^{-3}$ which is larger than the baryon

density. For such a dense condensate one would suspect that non-linear kaon self-interactions might be important. The order $\theta^{4}$ corrections to the thermodynamic potential are easily found from Eq. (56):

$$
\Delta \Omega_{4}=-\frac{f^{2} \theta^{4}}{24}\left(m_{K}^{2}-4 \mu^{2}\right)
$$

The result of adding this correction to $\Omega_{2}$ is shown as the dashed curve in Fig. 18. It greatly alters the behavior of $\Omega(\theta)$ for $\theta \gtrsim 1$. The exsistence of a second minimum suggests that a first order phase transition is still possible, but at larger $\mu_{n}$. However, we find that this is not the case and a second-order transition 
occurs at $\mu_{n}=1213 \mathrm{MeV}$. It is possible to incorporate all powers of $\theta$ arising from self-interactions in the chiral model. In this case the correction to the grand potential is

$$
\Delta \Omega_{n}=2 m_{K}^{2} f^{2} \sin ^{2} \frac{1}{2} \theta-\frac{1}{2} \mu^{2} f^{2} \sin ^{2} \theta-\frac{1}{2} f^{2} \theta^{2}\left(m_{K}^{2}-\mu^{2}\right) .
$$

The result of including this correction is shown as the dot-dashed curve in Fig. 18. In this case no first order phase transition is possible in the vicinity of $\mu_{n}=\mu_{c}$. Instead a second order phase transition occurs once again at $\mu_{n}=1213 \mathrm{MeV}$; this is because kaon self interactions play no role when $\theta$ is small. Despite our findings here, it is not clear if kaon self-interactions will generically disfavor a first order transition. This is because we have ignored higher order operators in the chiral expansion which will become important with increasing $\theta$. The indication from phenomenological chiral perturbation theory [37] is that such effects can be significant when $\theta \simeq 2$. The robust finding here is that the higher order kaon self-interactions predicted by the lowest order chiral Lagrangian lead to a second order, rather than a first order, phase transition. 


\section{REFERENCES}

[1] M. Prakash, I. Bombaci, M. Prakash, P.J. Ellis, J.M. Lattimer and R. Knorren, Phys. Rep. 280, 1 (1997).

[2] V. Thorsson, M. Prakash and J.M. Lattimer, Nucl. Phys. A 572, 693 (1994).

[3] W. Keil and H.T. Janka, Astron. and Astrophys. 296, 145 (1994).

[4] J.A. Pons, S. Reddy, M. Prakash, J.M. Lattimer and J.A. Miralles, Astrophys. J. 513, 780 (1999).

[5] N.K. Glendenning and J. Schaffner-Bielich, Phys. Rev. C 60, 025803 (1999).

[6] S. Reddy, G. Bertsch and M. Prakash, Phys. Lett. B 475, 1 (2000).

[7] R. Knorren, M. Prakash and P.J. Ellis, Phys. Rev. C 52, 3470 (1995).

[8] J. Schaffner and I.N. Mishustin, Phys. Rev. C 53, 1416 (1996).

[9] D. B. Kaplan and A. E. Nelson, Phys. Lett. B 175, 57 (1986); 179, 409 (1986) (E).

[10] H.D. Politzer and M.B. Wise, Phys. Lett. B 273, 156 (1991).

[11] G.E. Brown, K. Kubodera, M. Rho and V. Thorsson, Phys. Lett. B 291, 355 (1992).

[12] T. Maruyama, H. Fujii, T. Muto and T. Tatsumi, Phys. Lett. B 337, 19 (1994).

[13] T. Muto, T. Tatsumi and N. Iwamoto, Phys. Rev. D 61, 083002 (2000); ibid. D 61, 063001 (2000).

[14] T. Tatsumi and M. Yasuhira, Nucl. Phys. A 670, 218 (2000).

[15] B. D. Serot and J. D. Walecka, Advances in Nuclear Physics 19 ed. J.W. Negele and E. Vogt (Plenum, NY, 1986); B. D. Serot, Rep. Prog. Phys. 55, 1855 (1992).

[16] J. Boguta and A. Bodmer, Nucl. Phys. A292, 413 (1977).

[17] N.K. Glendenning and S.A. Moszkowski, Phys. Rev. Lett. 67, 2414 (1991).

[18] J. Zimanyi and S.A. Moszkowski, Phys. Rev. C 42, 1416 (1990).

[19] C.-H. Lee, S. Reddy and M. Prakash, Proc. of Int. Workshop XXVI on Gross Properties of Nuclei and Nuclear Excitations, ed. M. Buballa, W. Nörenberg, J. Wambach and A. Wirzba (Hirschegg, Austria, 1998) p. 86.

[20] V.I. Ogievetskij and I.V. Polubarinov, Ann. Phys. (NY) 25, 358 (1963).

[21] J. I. Kapusta, Finite Temperature Field Theory, Cambridge University Press, 1985.

[22] V. Thorsson and P.J. Ellis, Phys. Rev. D 55, 5177 (1997); T.Tatsumi and M. Yasuhira, Phys. Lett. B 441, 9 (1998) and Nucl. Phys. A 653, 133 (1999).

[23] N.K. Glendenning, Nucl. Phys. B (Proc. Suppl.) 24B, 110 (1991); Phys. Rev. D 46, 1274 (1992).

[24] T.E.O. Ericson and F. Scheck, Nucl. Phys. B19, 450 (1970).

[25] E. Friedman, A. Gal and C.J. Batty, Nucl. Phys. A 579, 578 (1994); E. Friedman, A. Gal, J. Mareš and A. Cieplý, Phys. Rev. C 60, 024314 (1999).

[26] T. Waas and W. Weise, Nucl. Phys. A 625, 287 (1997).

[27] A. Ramos and E. Oset, Nucl. Phys. A 671, 481 (2000).

[28] A. Baca, C. García-Recio, and J. Nieves, nucl-th/0001060.

[29] J. W. Negele and D. Vautherin, Nucl. Phys. A 207, 298 (1974).

[30] G. Baym, C. J. Pethick and J. Sutherland, Astrophys. J. 170, 299 (1971).

[31] V.R. Pandharipande, C.J. Pethick, and V. Thorsson, Phys. Rev. Lett. 75, 4567 (1995).

[32] J. Carlson, H. Heiselberg, and V.R. Pandharipande, nucl-th/9912043.

[33] J.M. Lattimer, C.J. Pethick, M. Prakash, and P. Haensel, Phys. Rev. Lett. 66, 66 (1991).

[34] A. Akmal, V.R. Pandharipande, and D. G. Ravenhall, Phys. Rev. C 58, 1804 (1998).

[35] L. Engvik, M. Hjorth-Jensen, E. Osnes, G. Bao, and E. Østgaard, Phys. Rev. Lett. 73, 2650 (1994); Astrophys. Jl. 469, 794 (1996).

[36] S.M. Johns, P.J. Ellis and J.M. Lattimer, Astrophys. J. 473, 1020 (1996).

[37] J. Gasser and H. Leutwyler, Nucl. Phys. B250, 465 (1985). 
TABLE I. Meson-nucleon coupling constants fitted to a binding energy/particle of $16.3 \mathrm{MeV}$ at an equilibrium density of $n_{0}=0.153 \mathrm{fm}^{-3}$ in nuclear matter with a compression modulus $K=240 \mathrm{MeV}$ and effective mass $M^{*}=0.78 M$. The symmetry energy coefficient $a_{\text {sym }}$ is $32.5 \mathrm{MeV}$.

\begin{tabular}{|c|c|c|c|c|c|}
\hline Model & $\begin{array}{c}\frac{g_{\sigma}}{m_{\sigma}} \\
(\mathrm{fm})\end{array}$ & $\begin{array}{c}\frac{g_{\omega}}{m_{\omega}} \\
(\mathrm{fm})\end{array}$ & $\begin{array}{c}\frac{g_{\rho}}{m_{\rho}} \\
(\mathrm{fm})\end{array}$ & $b$ & $c$ \\
\hline GM & 3.1507 & 2.1954 & 2.1888 & 0.008659 & -0.002421 \\
\hline ZM & 3.1228 & 2.1954 & 2.1888 & -0.006418 & 0.002968 \\
\hline
\end{tabular}

TABLE II. Kaon optical potentials for the models GS and TW. The values in the second column refers to the linear approximation of the exact results for the models GS and TW shown in the third and fourth columns. All results in this paper have been labelled according to the linear approximation (in order to make a comparison with the results of GS), which is exact for the model KPE.

\begin{tabular}{r|rrr}
\hline \hline$g_{\sigma K} \sigma$ & $-U_{K}^{\operatorname{lin}}=-U_{K}^{K P E}$ & $-U_{K}^{G S}$ & $-U_{K}^{T W}$ \\
\hline 63 & 80 & 81 & 80 \\
103 & 100 & 100 & 95 \\
143 & 120 & 117 & 109 \\
183 & 140 & 136 & 122 \\
223 & 160 & 150 & 134 \\
\hline \hline
\end{tabular}




\section{FIGURE CAPTIONS}

FIG. 1: Pressure versus baryon number density for the six choices of the nucleon and kaon Lagrangians considered in this paper. The temperature $T=0$ and there are no trapped neutrinos $\left(Y_{\nu}=0\right)$. Selected values for the kaon optical potential $U_{K}^{\text {lin }}$ are indicated. The solid lines show the pressure in the pure phases I (nucleons only) and II (the high-density nucleon-kaon condensed phase). The dashed lines show the pressures obtained by imposing Gibbs' criteria for phase equilibrium in a mixed-phase region for the case of first order transitions. For the KPE choice of the kaon Lagrangian, Gibbs' criteria could not be satisfied despite the occurence of first order phase transitions in some cases.

FIG. 2: The density dependences of the scalar, vector, and iso-vector fields for different choices of the nucleon and kaon Lagrangians $\left(T=0, Y_{\nu}=0\right)$. The solid curves show the chemical potential $\mu_{n}-\mu_{p}=\mu$. In this figure, results are shown only for the pure phases I and II; the mixed phase produced by satisfying Gibbs' criteria is ignored.

FIG. 3: As for Fig. 2, but for the density dependences of the kaon and nucleon effective masses. The solid curves show the condensate amplitude.

FIG. \&: The electron chemical potential $\mu$ versus the neutron chemical potential $\mu_{n}$ in pure phases I and II, and in the mixed phase. The pure phase I (solid curve) consists of nucleons and leptons. The pure phase II (dashed curve) is comprised of a kaon condensate coexisting with nucleons and leptons. The mixed phase (dots) is constructed by satisfying Gibbs' rules for phase equilibrium.

FIG. 5: Individual charge densities of pure phases I and II and the volume fraction $\chi$ of phase I in the mixed phase as a function of baryon density. Results are for the GM+GS model with $U_{K}^{\operatorname{lin}}=-120 \mathrm{MeV}$ and for ZM+TW model with $U_{K}^{\text {lin }}=-140 \mathrm{MeV}$.

FIG. 6: The density dependences of the scalar, vector, and iso-vector fields for two choices of the nucleon and kaon Lagrangians $\left(T=0, Y_{\nu}=0\right)$. Phase $\mathrm{I}$ is the pure nucleon phase and phase II is the high-density nucleon-kaon condensed phase. The vertical lines demark the mixed phase region.

FIG. 7: The density dependences of the chemical potential $\mu_{n}-\mu_{p}=\mu$, the kaon (K) and nucleon $(\mathrm{N})$ effective masses, and the condensate amplitude $\left(T=0, Y_{\nu}=0\right)$. Notation is as in Fig. 6 .

FIG. 8: The electron chemical potential $\mu$ in phase II matter versus charge density for different models at a fixed neutron chemical potential of $\mu_{n}=1250 \mathrm{MeV}$. In all cases, the optical potential $U_{K}^{\text {lin }}=-120$ $\mathrm{MeV}$.

FIG. 9: Left panel: The gravitational mass as a function of the central baryon number density for

the cases GM+GS and ZM+TW $\left(T=0, Y_{\nu}=0\right)$. Curves are labelled by the values of $U_{K}^{\operatorname{lin}}$ and the EOS includes a mixed phase region. Right panel: The gravitational mass as a function of the stellar radius.

FIG 10: The density dependences of the kaon energy $\omega$ in matter with different isospin content. The top panel compares results of GM+KPE, GM+GS and ZM+TW models for beta stable neutron star matter for $U_{K}^{\text {lin }}=-80$ and $-120 \mathrm{MeV}$, respectively. The bottom panel shows results for the $\mathrm{ZM}+\mathrm{TW}$ model with $U_{K}^{\operatorname{lin}}=-80 \mathrm{MeV}$ in pure neutron matter, beta stable neutron star matter and nuclear matter.

FIG 11: The relative concentrations of hadrons and leptons as functions of baryon number density 
for three representative snapshots during the evolution of a PNS. The results shown are for the model GM+GS. To the left of the vertical line there is no kaon condensate, to the right a mixed phase is present.

FIG 12: Same as Fig. 11, but for the model ZM+TW.

FIG 13: The pressure versus baryon number density for three representative snapshots during the evolution of a PNS. The cases shown in the upper panels produce only second order phase transitions. For the cases in the lower panels the transitions are first order, except for ZM+TW with $s>0$. In the lower panels, heavy curves include a mixed phase region and light curves ignore a mixed phase region.

FIG 14. The temperature as a function of baryon density for two snapshots during the PNS evolution. Other notation is as in Fig. 12.

FIG 15: The phase diagram of kaon condensed matter for the case GM+GS and $U_{K}^{\operatorname{lin}}=-120 \mathrm{MeV}$. The left panel shows results at zero temperature in the density versus lepton concentration plane. The dashed curve shows the minimum lepton concentration for each density, which occurs for trapped neutrino concentration $Y_{\nu}=0$. The right panel shows results in the density versus temperature plane for neutrino free matter $\left(Y_{\nu}=0\right)$.

FIG 16: Same as Fig. 15, but for the model ZM+TW and $U_{K}^{\operatorname{lin}}=-140 \mathrm{MeV}$.

FIG 17. The gravitational mass versus central baryon number density in the GM+GS and ZM+TW models for three representative snapshots during the PNS evolution.

FIG 18. The thermodynamic potential as a function of the condensate order parameter $\theta$. Results are shown for the GM+GS model near the critical density $\left(\mu_{n} \simeq 1160 \mathrm{MeV}\right.$ and $\left.\mu \simeq 243 \mathrm{MeV}\right)$ with optical potential $U_{K}^{\operatorname{lin}}=-120 \mathrm{MeV}$. 


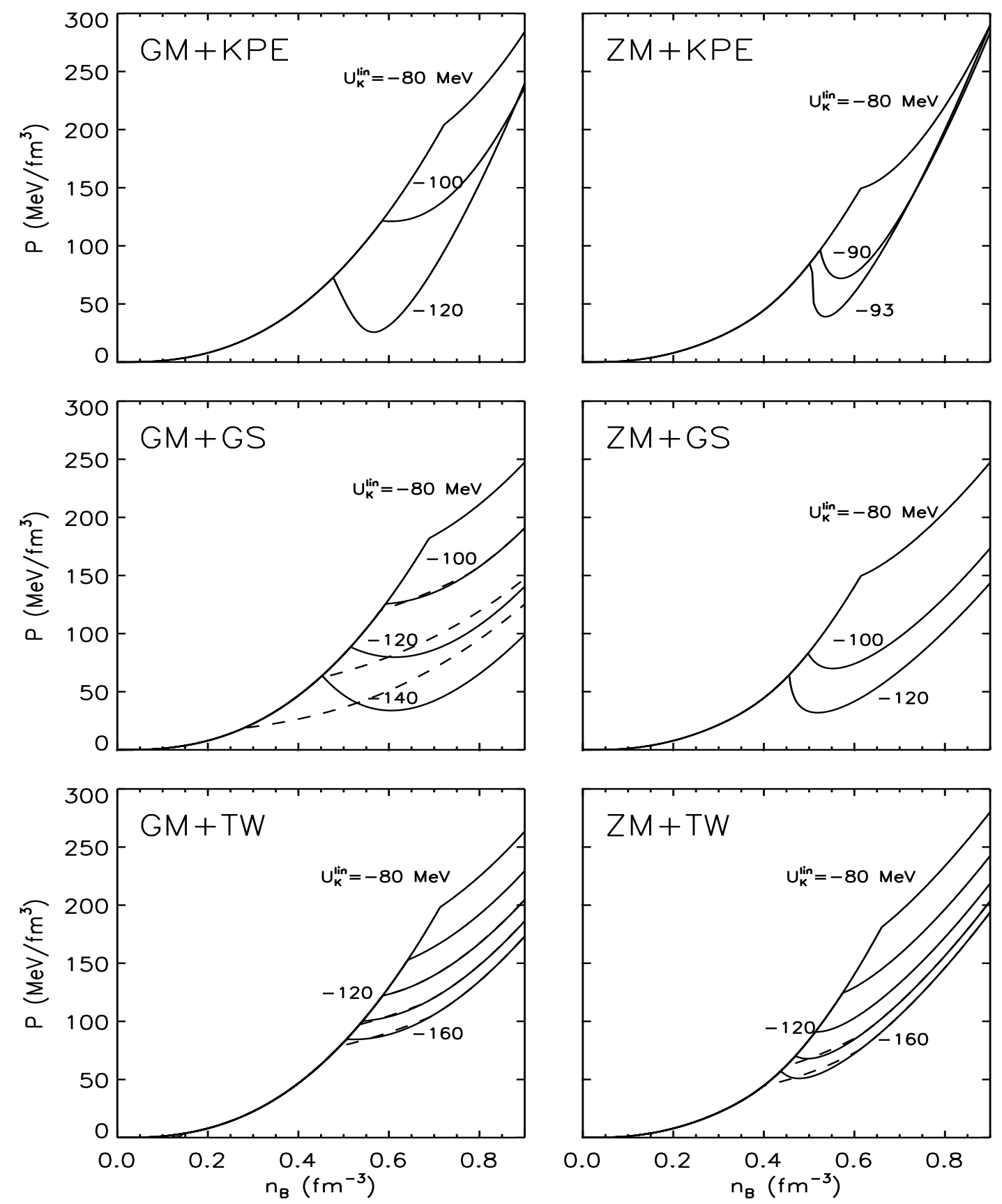

FIG. 1. 

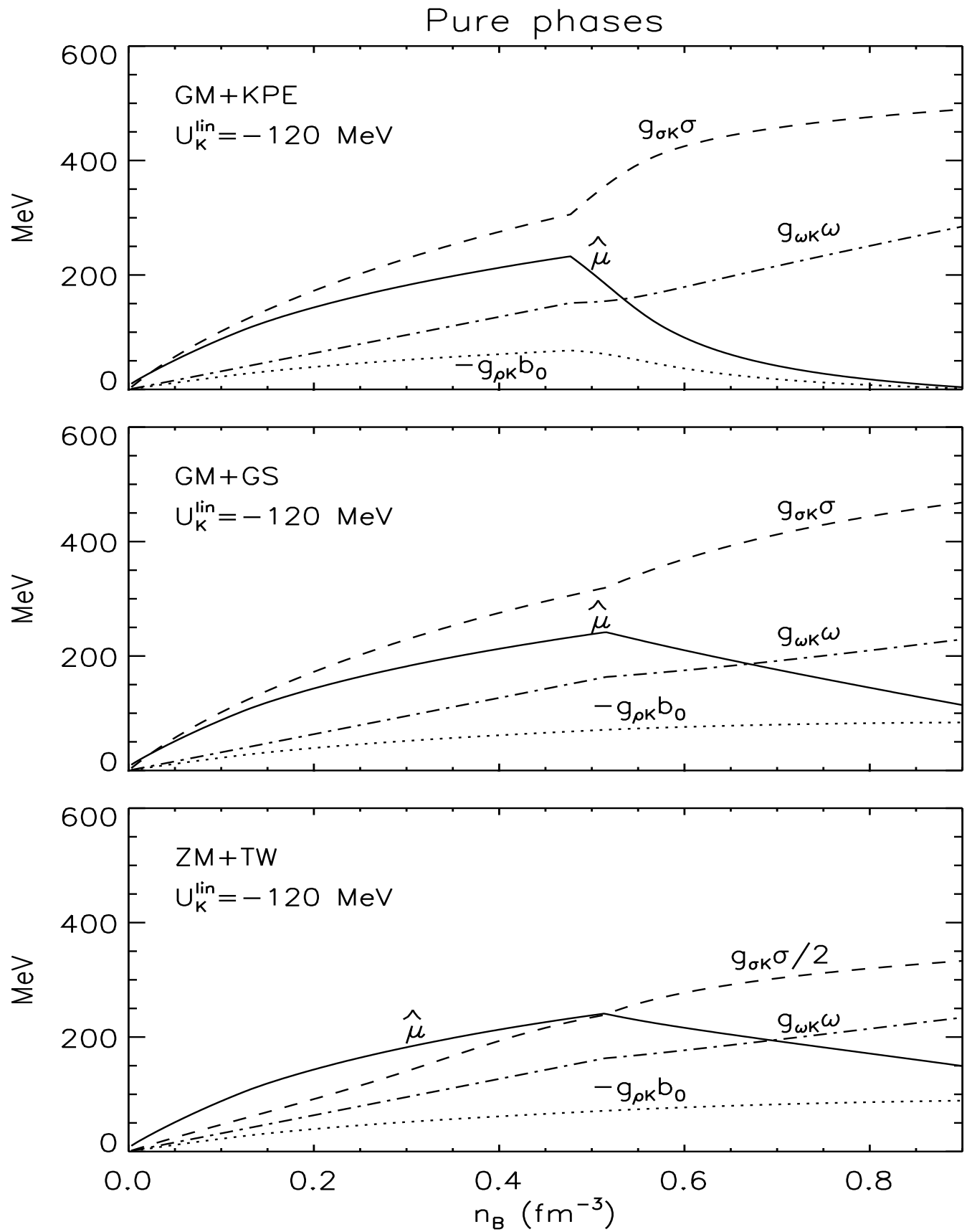

FIG. 2. 

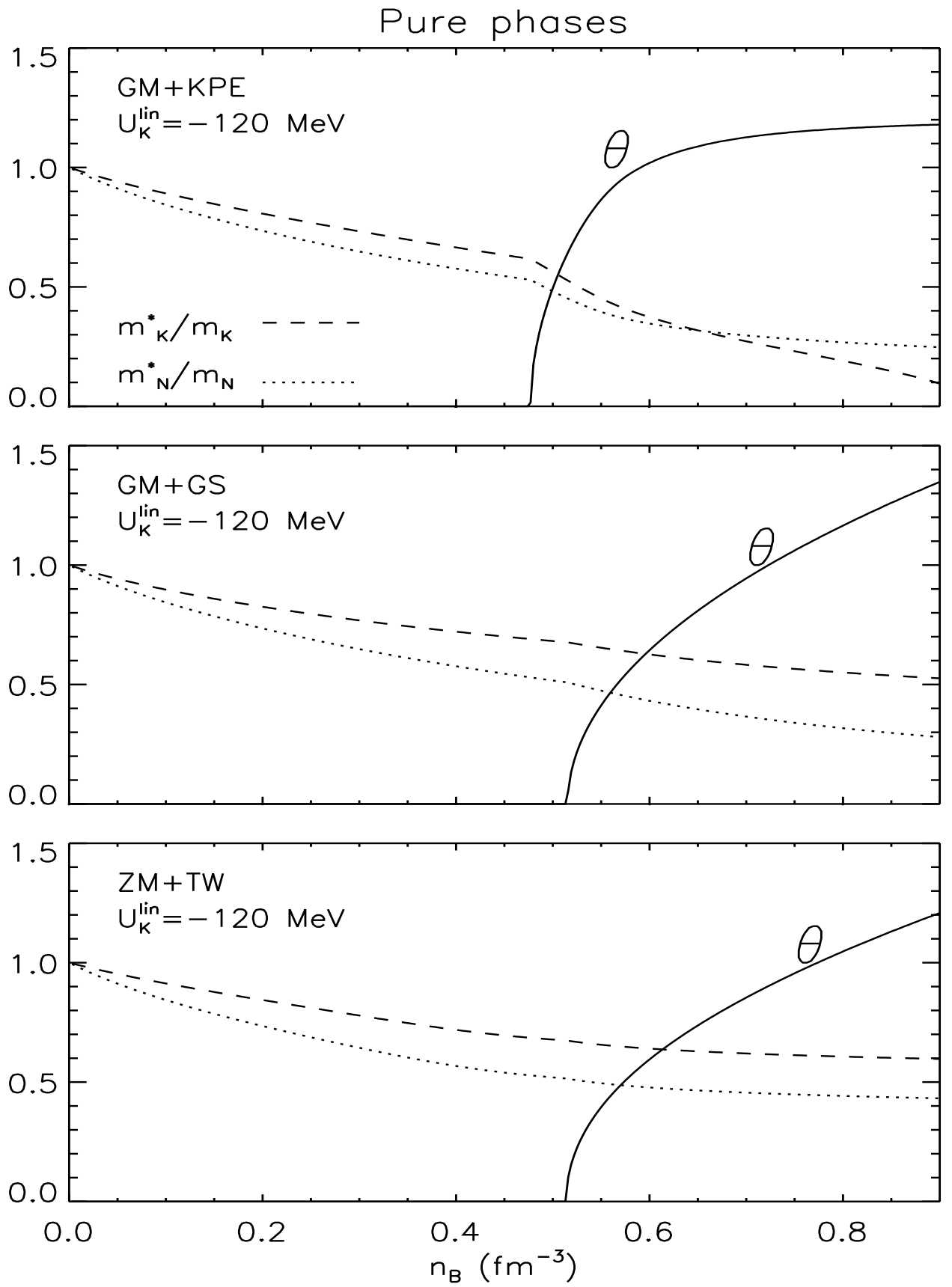

FIG. 3. 

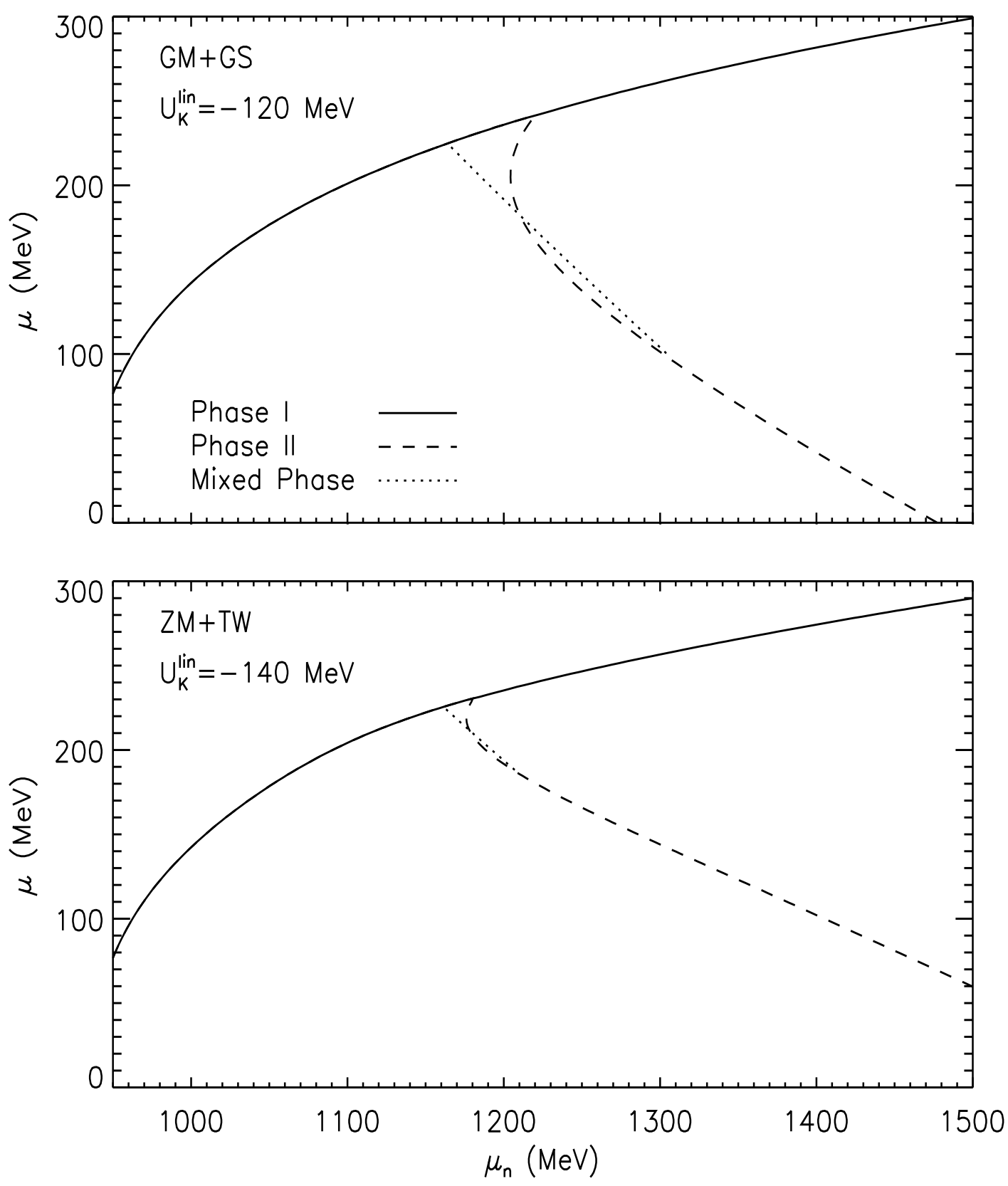

FIG. 4. 

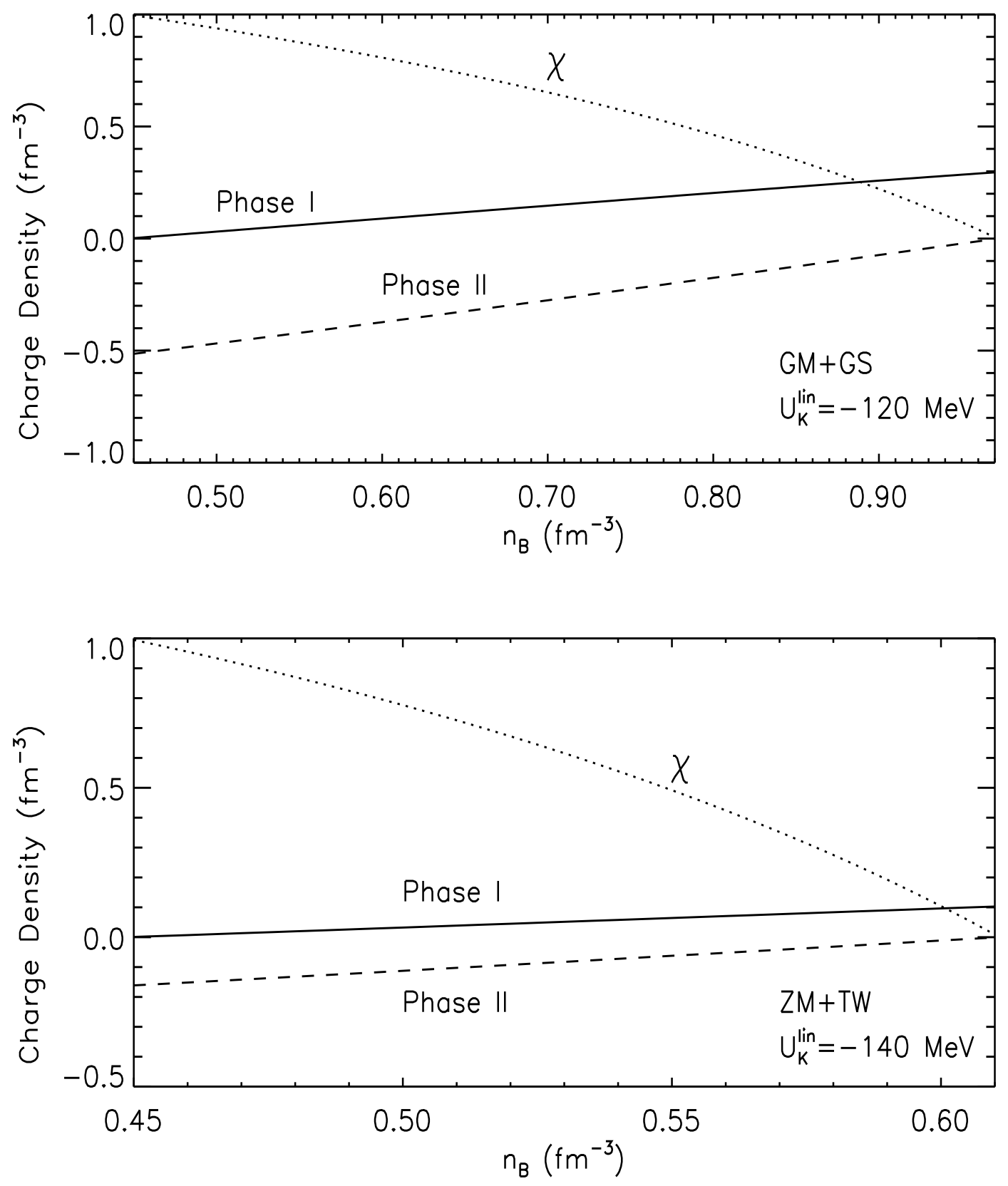

FIG. 5. 

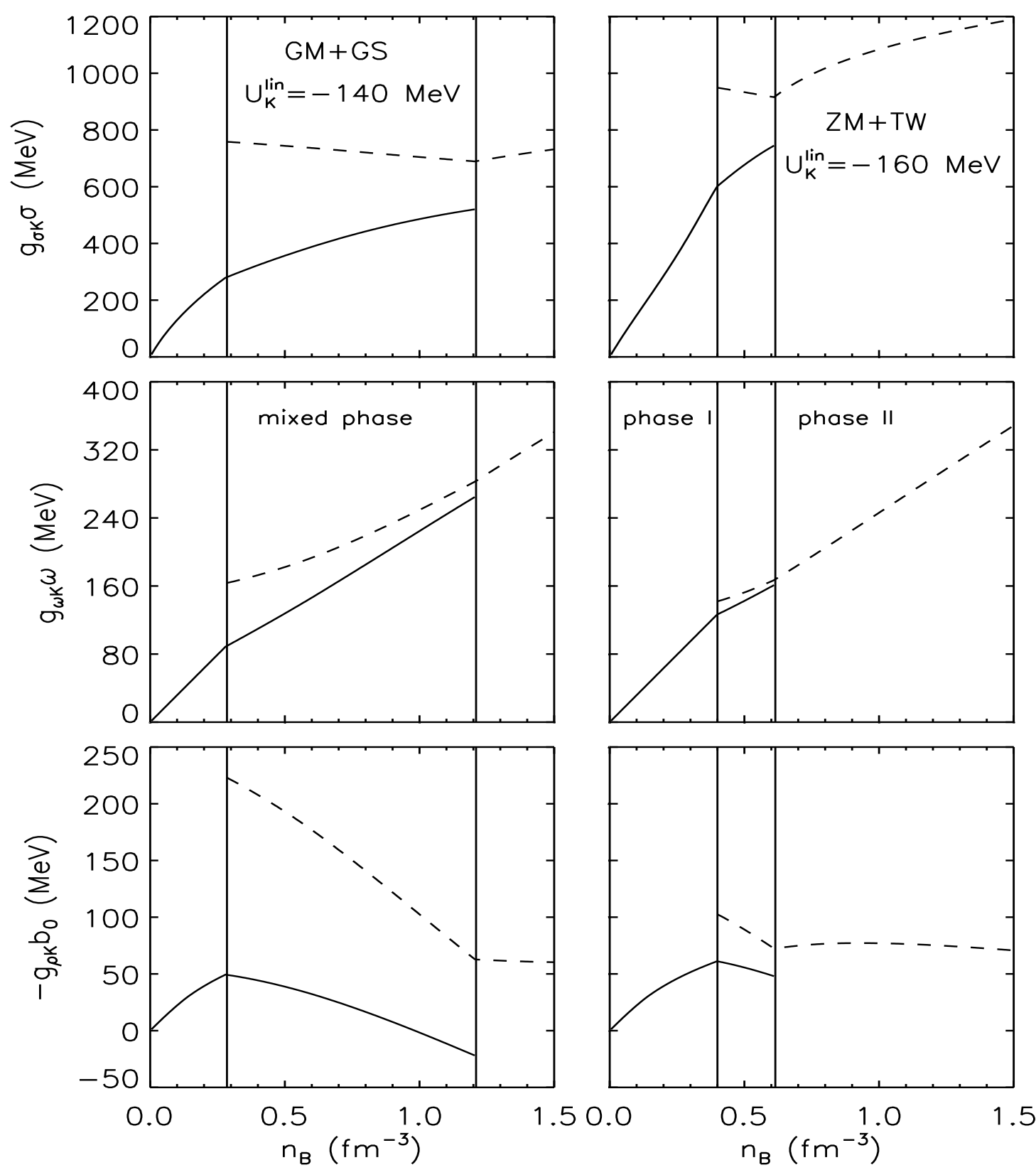

FIG. 6. 


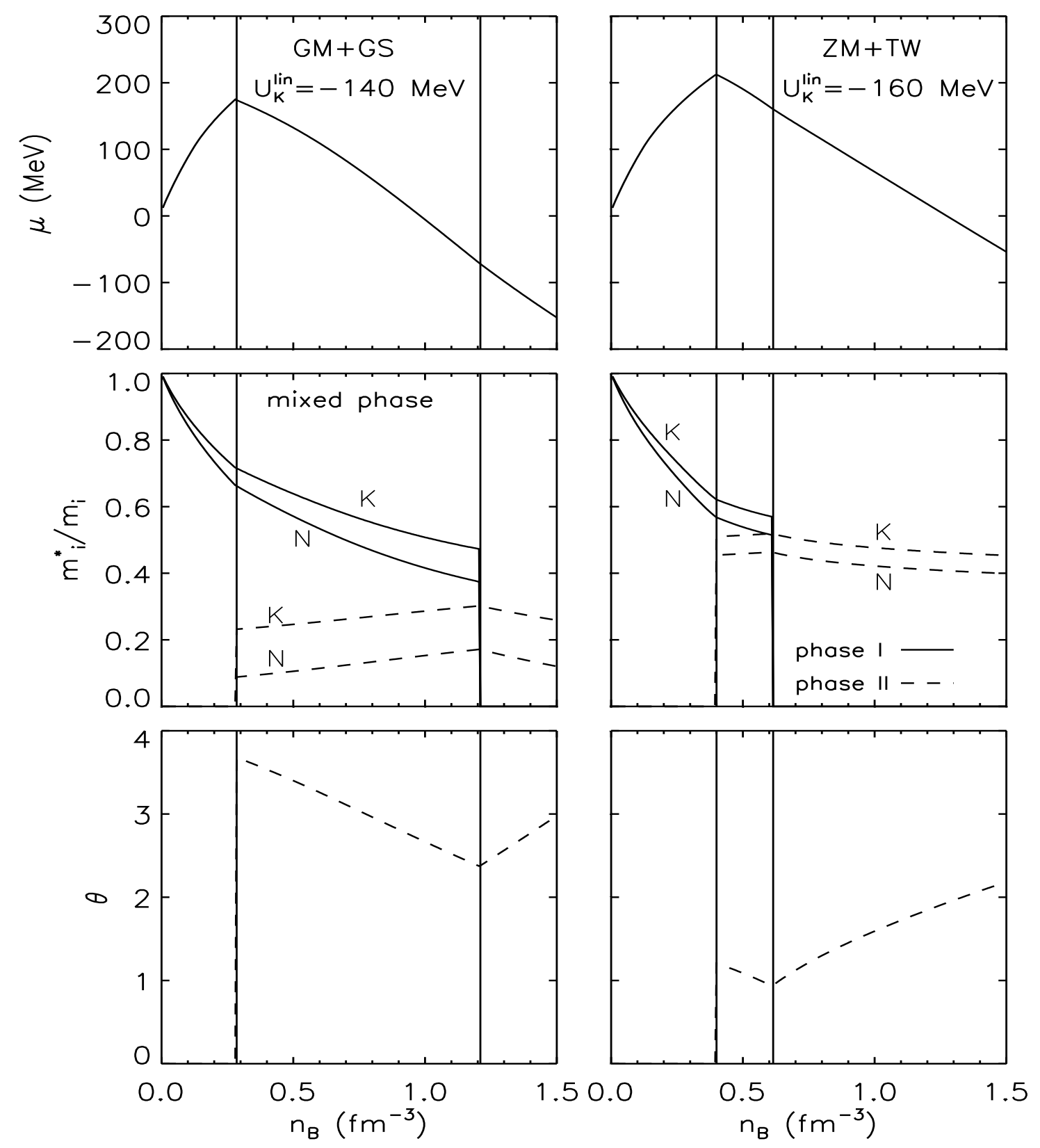

FIG. 7. 


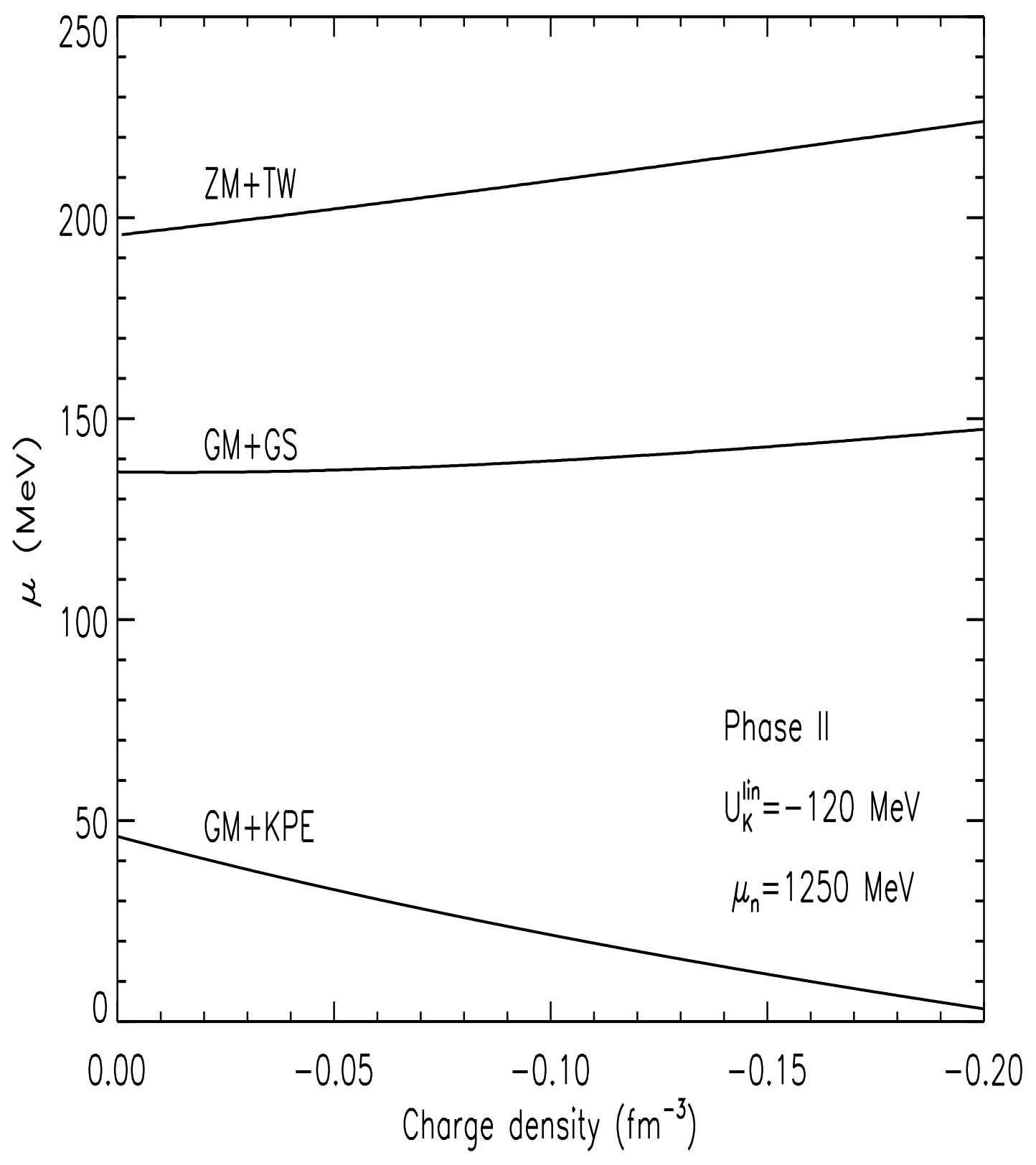

FIG. 8. 

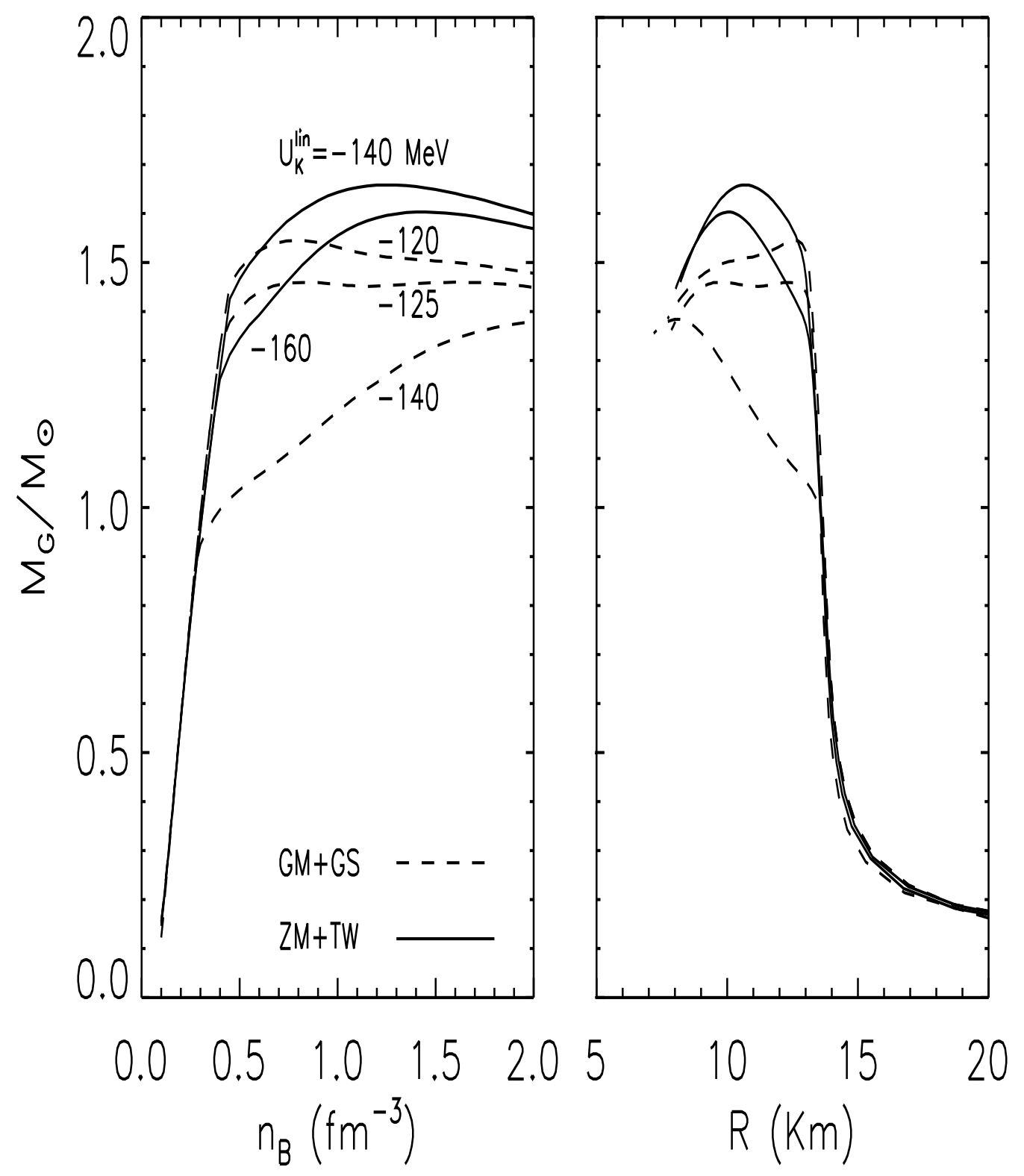

FIG. 9. 

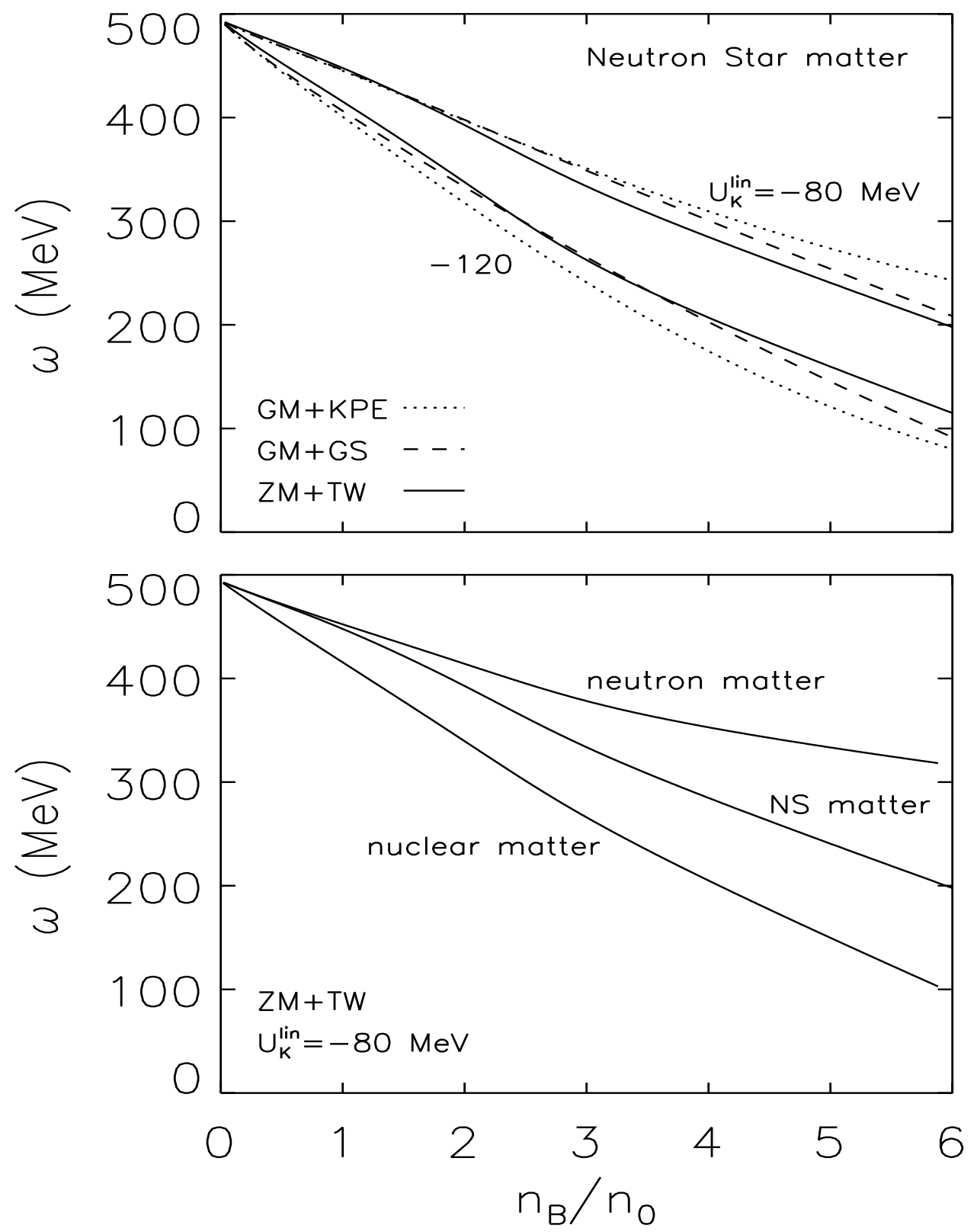

FIG. 10. 

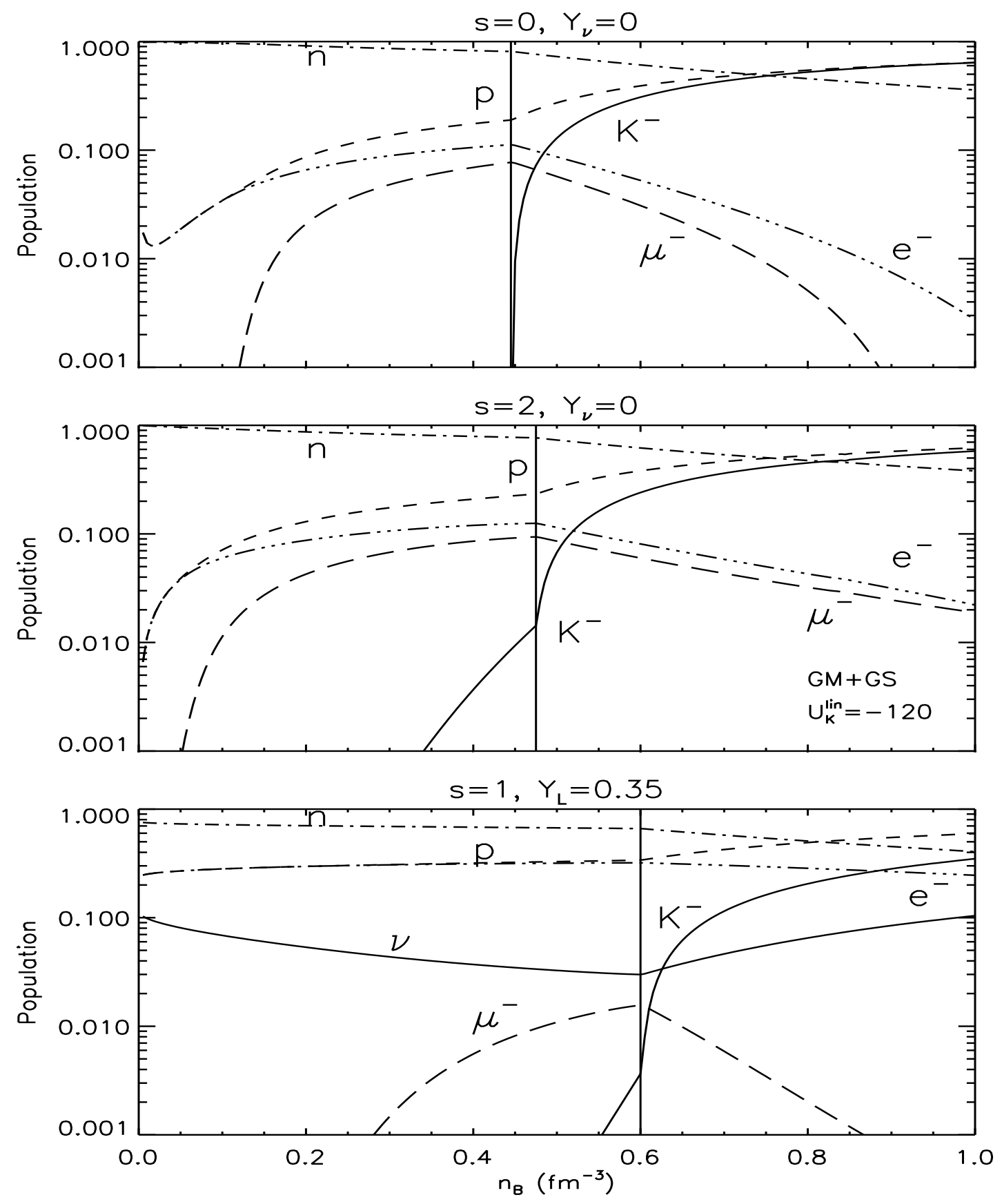

FIG. 11. 

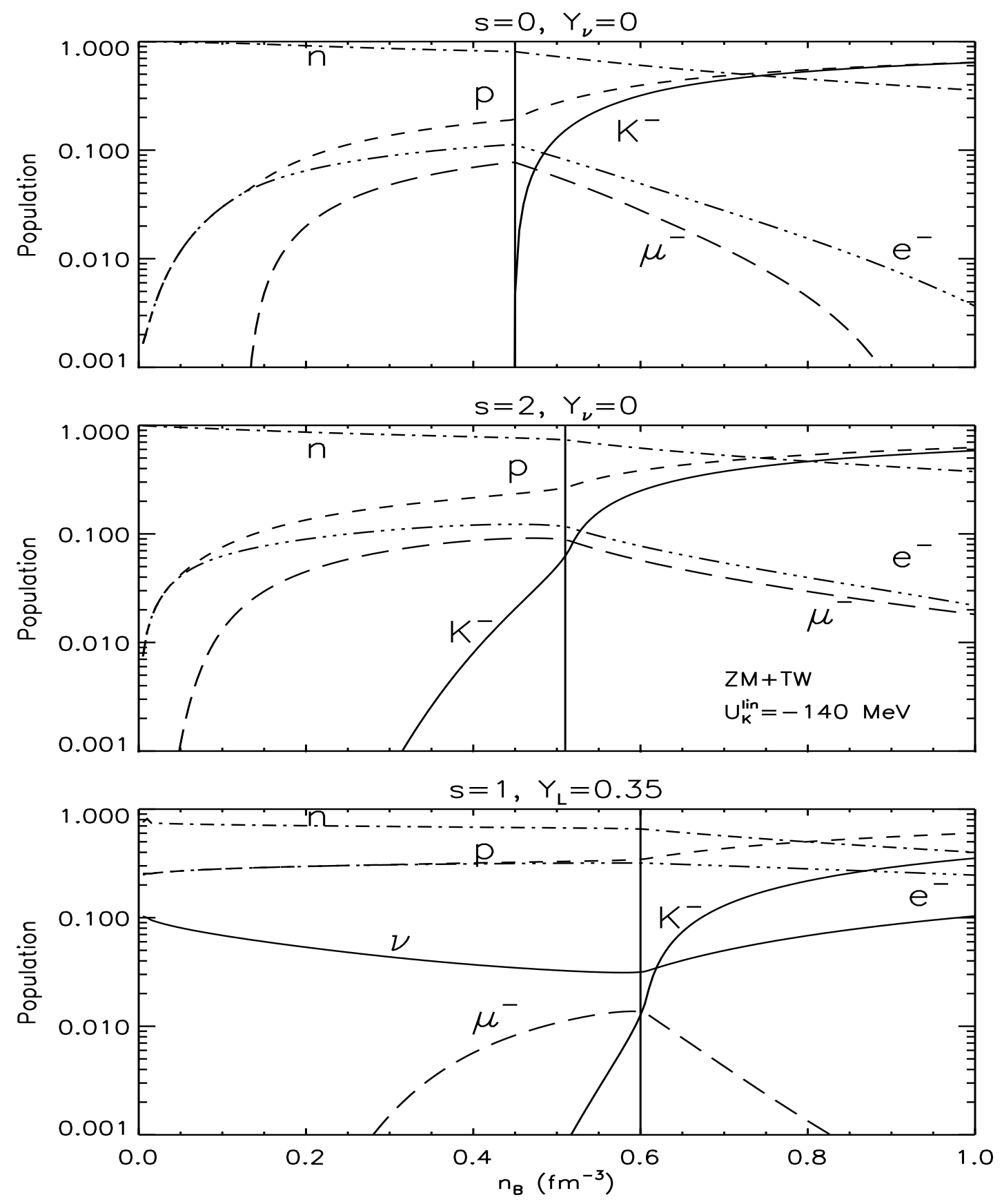

FIG. 12. 

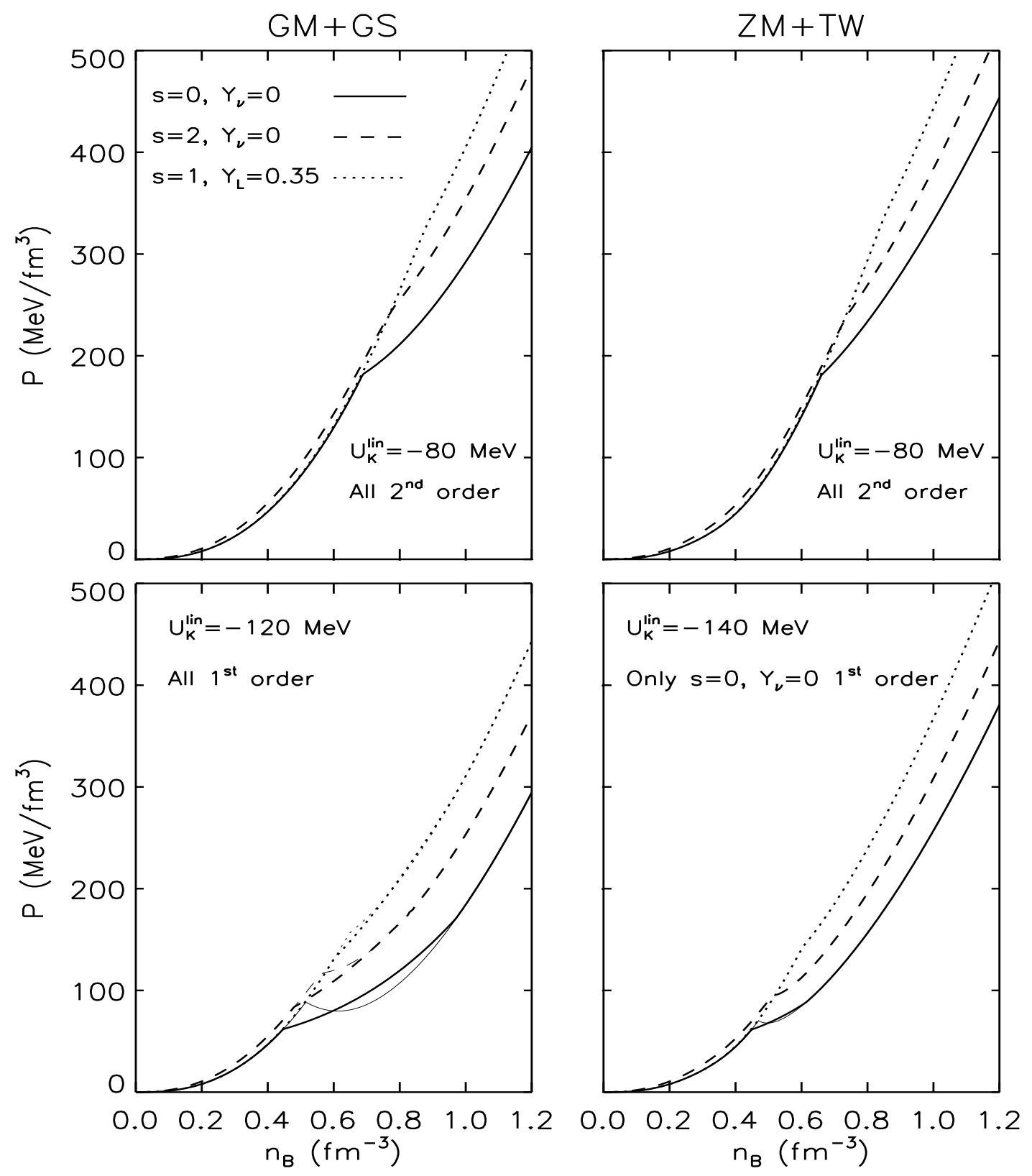

FIG. 13. 

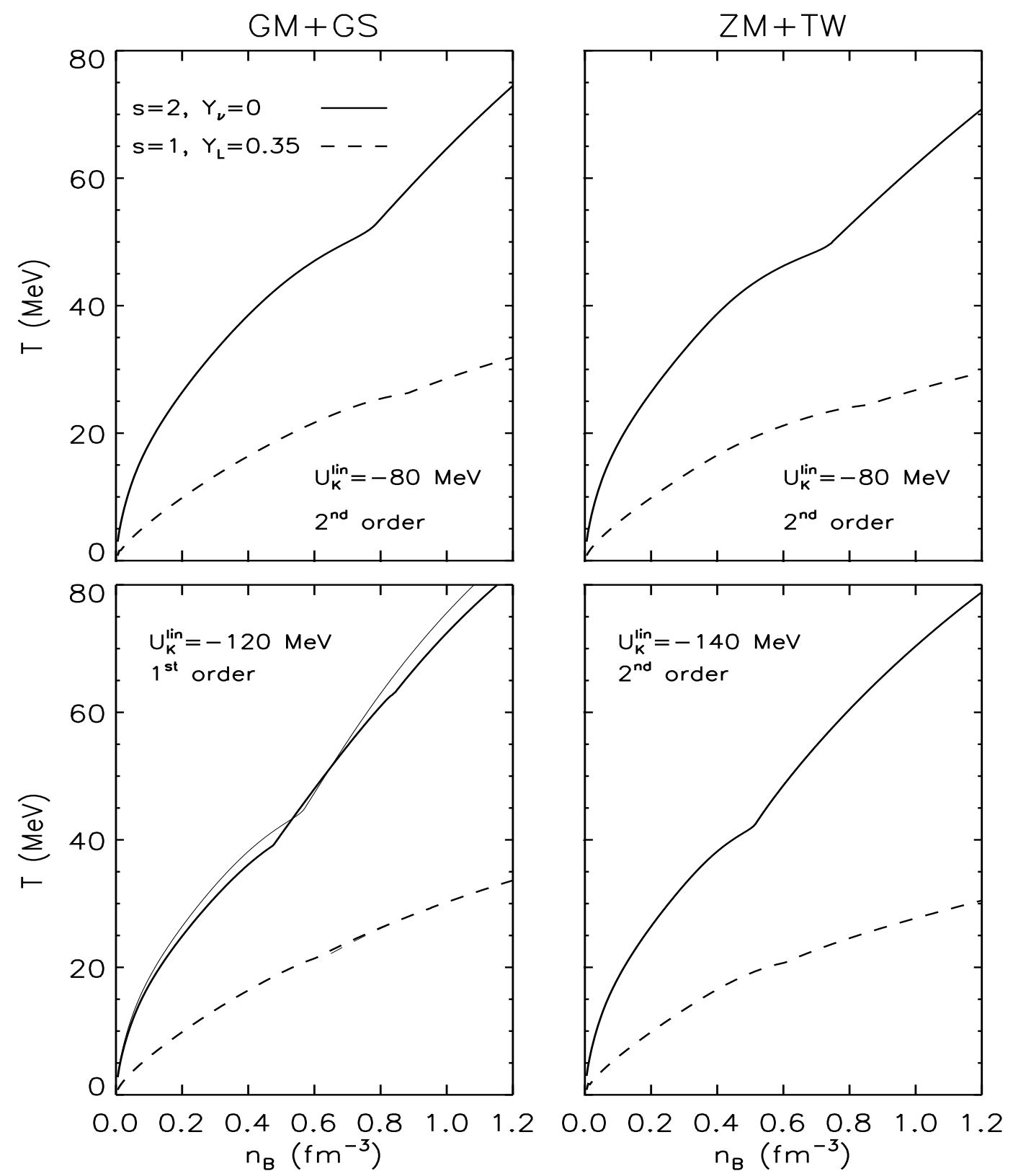

FIG. 14. 

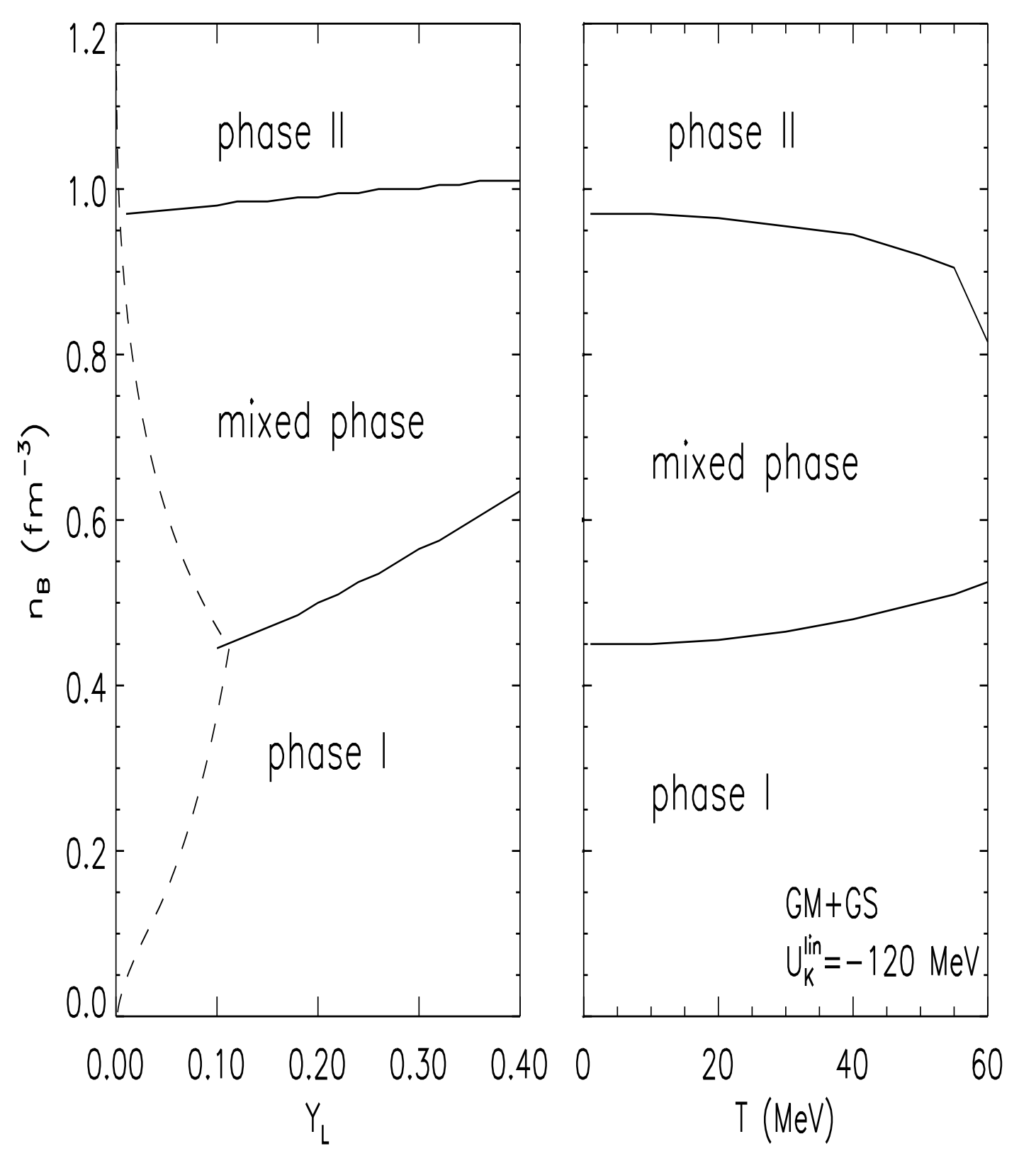

FIG. 15. 

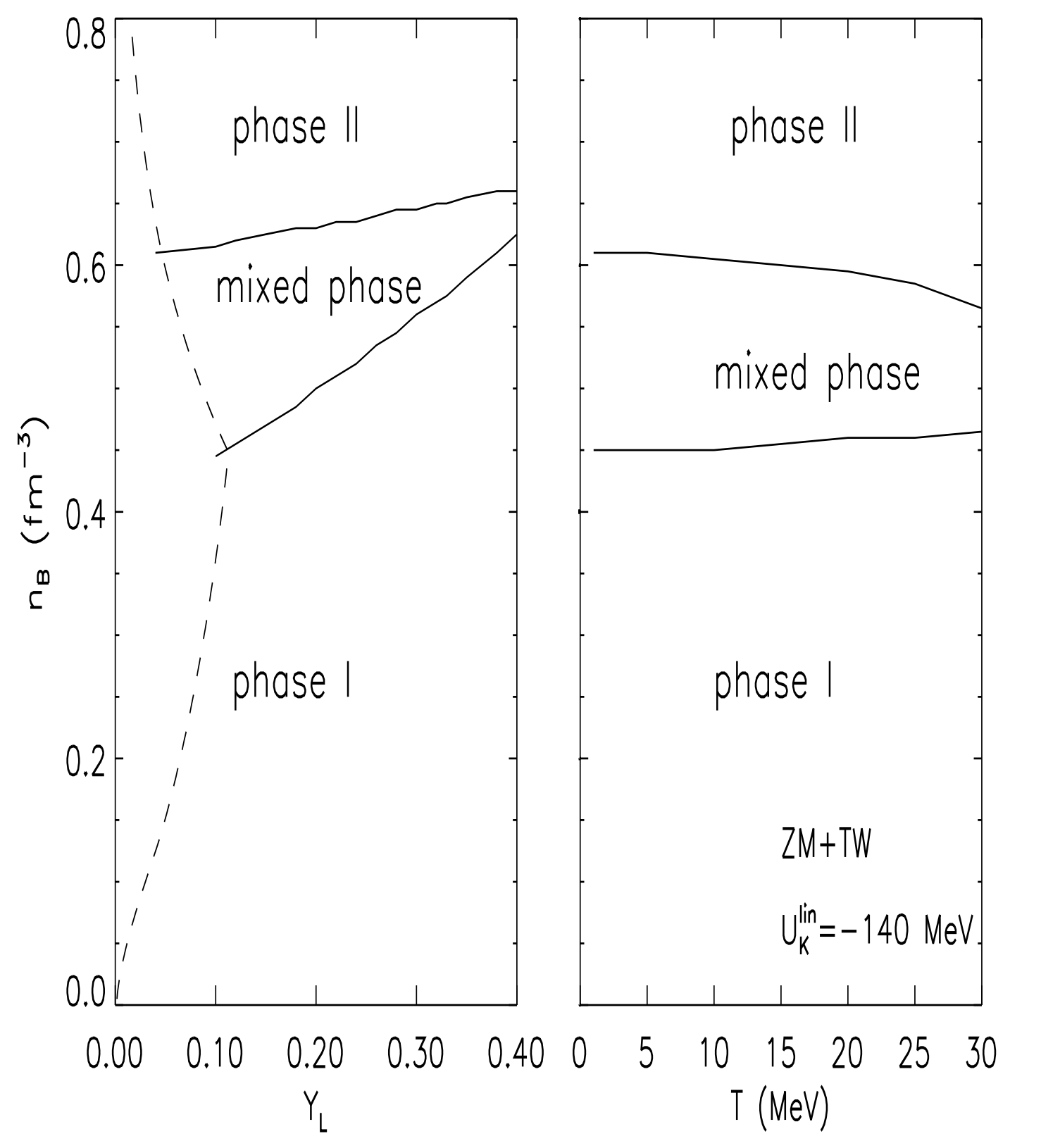

FIG. 16. 

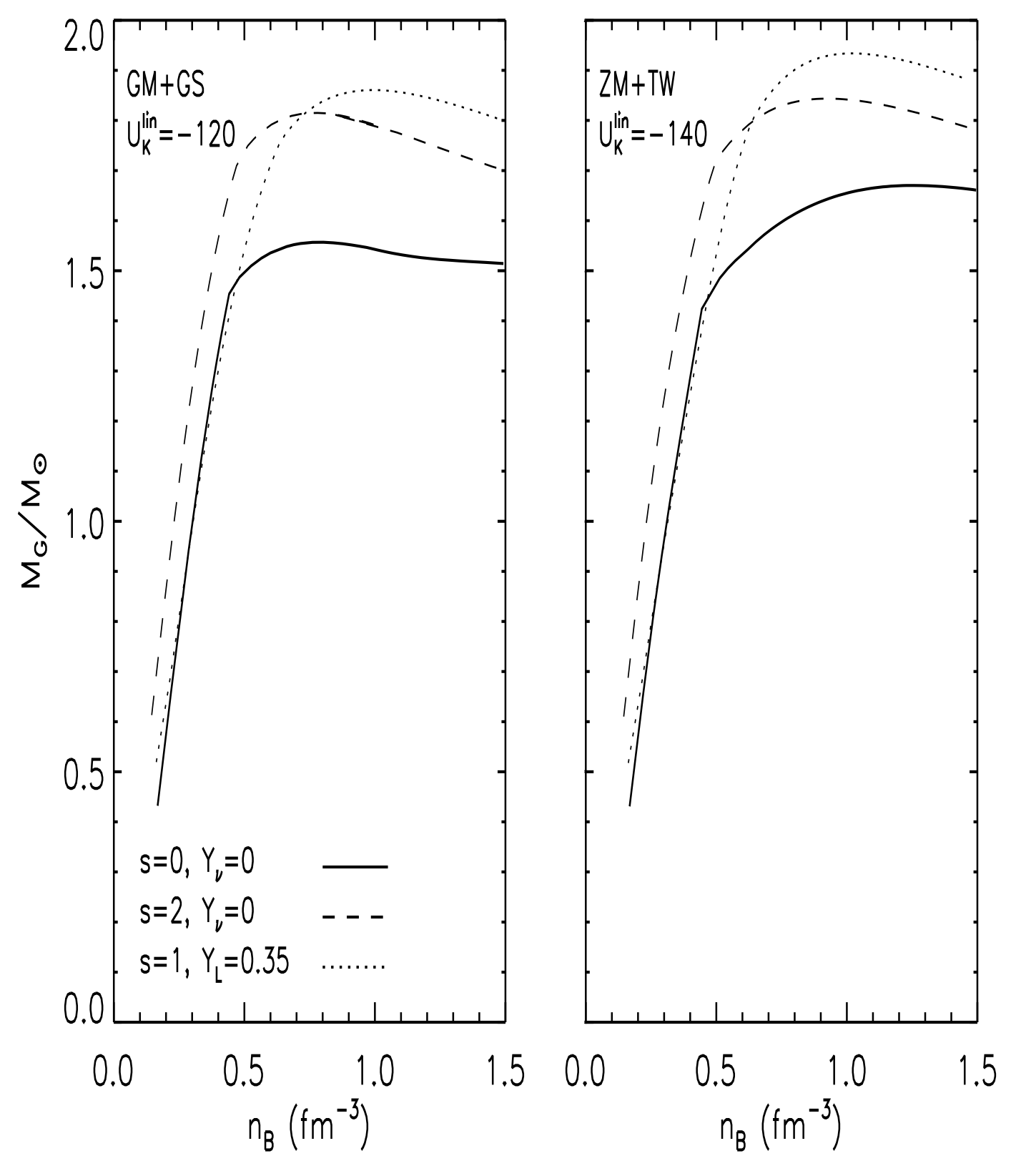

FIG. 17. 


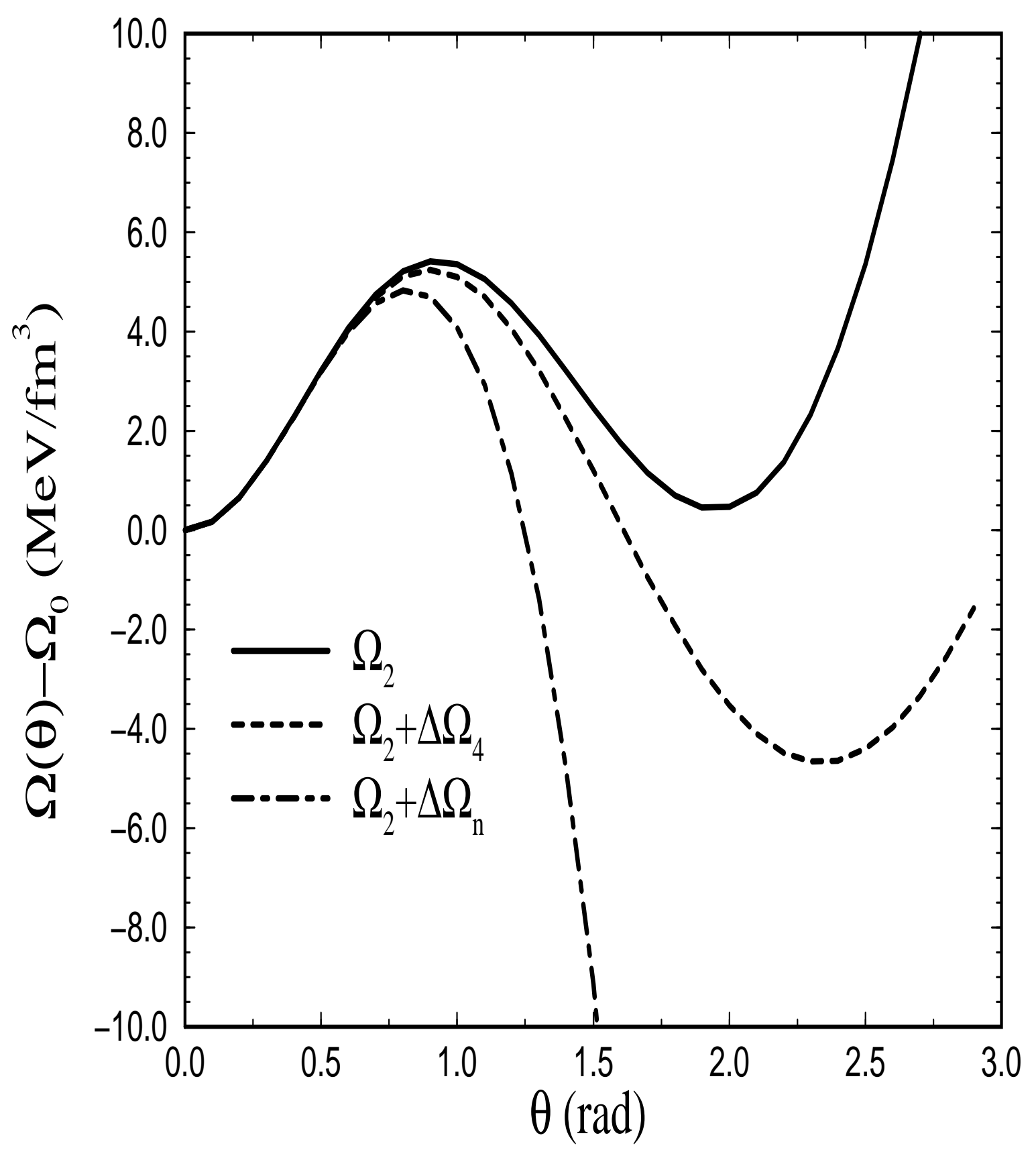

FIG. 18. 